\title{
Transitional regimes of natural convection in a differentially heated cubical cavity under the effects of wall and molecular gas radiation
}

\author{
L. Soucasse,,$^{1,2}$ Ph. Rivière, ${ }^{1,2}$ A. Soufiani, ${ }^{1,2, a)}$ S. Xin, ${ }^{3}$ and P. Le Quéré ${ }^{4}$ \\ ${ }^{1}$ CNRS, UPR 288, Laboratoire EM2C, 92290 Châtenay-Malabry, France \\ ${ }^{2}$ École Centrale Paris, 92290 Châtenay-Malabry, France \\ ${ }^{3}$ CNRS/INSA-Lyon, UMR 5008, CETHIL, 69621 Villeurbanne, France \\ ${ }^{4}$ CNRS, UPR 3251, LIMSI, 91403 Orsay Cedex, France
}

(Received 11 October 2013; accepted 20 January 2014; published online 12 February 2014)

The transition to unsteadiness and the dynamics of weakly turbulent natural convection, coupled to wall or gas radiation in a differentially heated cubical cavity with adiabatic lateral walls, are studied numerically. The working fluid is air with small contents of water vapor and carbon dioxide whose infrared spectral radiative properties are modelled by the absorption distribution function model. A pseudo spectral Chebyshev collocation method is used to solve the flow field equations and is coupled to a direct ray tracing method for radiation transport. Flow structures are identified by means of either the proper orthogonal decomposition or the dynamic mode decomposition methods. We first retrieve the classical mechanism of transition to unsteadiness without radiation, characterized by counter-rotating streamwise-oriented vortices generated at the exit of the vertical boundary layers. Wall radiation through a transparent medium leads to a homogenization of lateral wall temperatures and the resulting transition mechanism is similar to that obtained with perfectly conducting lateral walls. The transition is due to an unstable stratification upstream the vertical boundary layers and is characterized by periodically oscillating transverse rolls of axis perpendicular to the main flow. When molecular gas radiation is accounted for, no periodic solution is found and the transition to unsteadiness displays complex structures with chimneys-like rolls whose axes are again parallel to the main flow. The origin of this instability is probably due to centrifugal forces, as suggested previously for the case without radiation. Above the transition to unsteadiness, at $\mathrm{Ra}=3 \times 10^{8}$, it is shown that both wall and gas radiation significantly intensify turbulent fluctuations, decrease the thermal stratification in the core of the cavity, and increase the global circulation. (c) 2014 AIP Publishing LLC. [http://dx.doi.org/10.1063/1.4864265]

\section{INTRODUCTION}

Radiation effects on natural convection have been studied since a long time due to their relevance to atmospheric physics ${ }^{1,2}$ or to engineering applications involving cavities. ${ }^{3,4}$ Wall and medium radiation were soon recognized as important mechanisms since they either modify the energy balance at the boundaries, or the local energy balance of the flowing medium. The numerical or analytical treatment of radiation transport in unsteady flows remains however a challenge due to its dependence on the propagation direction, frequency for radiating gases, in addition to time and space. For these reasons, and to the best of our knowledge, the transition to turbulence in 3D natural convection flows of radiating molecular gases has not been studied yet.

Transition to unsteadiness in differentially heated cavities without radiation has been investigated either using stability analyses or numerical simulations. Many studies have been devoted during the

\footnotetext{
a) Author to whom correspondence should be addressed. Electronic mail: anouar.soufiani@ecp.fr
} 
last three decades to $2 \mathrm{D}$ rectangular enclosures (see Refs. 5-8 among others). We only summarize here the studies related to 3D instabilities, for which different physical mechanisms have been identified when considering either adiabatic or conducting horizontal and lateral walls. We also focus on studies related to air flows with a Prandtl number close to 0.7.

For adiabatic side walls, Henkes and Le Quéré ${ }^{9}$ investigated the stability of 2D steady or unsteady base flows with respect to 3D periodic perturbations. They used time integration of linearized Navier-Stokes (NS) equations as well as full nonlinear time integrations. They showed that 3D perturbations were less stable than 2D perturbations leading to a lower critical Rayleigh number (Ra). Transition for adiabatic walls was detected between $\mathrm{Ra}=10^{7}$ and $10^{8}$. The origin was attributed to combined thermal and hydrodynamic effects. The critical Rayleigh number obtained with periodic 3D perturbations of 2D base flow was given in a more recent study by Xin and Le Quéré. ${ }^{10}$ An unstable steady mode was found around $\mathrm{Ra}=1.55 \times 10^{7}$, followed by unsteady instability between 4 and $4.5 \times 10^{7}$. Janssen and Henkes ${ }^{11}$ carried out 3D numerical simulations, restricted to the symmetries satisfied by NS equations and boundary conditions, to study the first instabilities appearing in a cubical cavity filled with air. They found a first transition from steady, almost-2D flow, to steady 3D flows, followed by a low-frequency unsteady instability. The occurrence of streamwise counter-rotating vortices in the curved flow regions at the exit of the vertical boundary layers let them suggest that this 3D instability is probably caused by centrifugal forces, similar to the Dean instability. Labrosse et al. ${ }^{12}$ released the symmetry assumptions made by Janssen and Henkes ${ }^{11}$ and carried out full 3D numerical simulations. They showed that first transition to unsteady flow in a cubic cavity with adiabatic walls occurs at a Rayleigh number between 3.1 and $3.5 \times 10^{7}, 6$ times lower than the corresponding $2 \mathrm{D}$ bifurcation. The unsteady instability was either a Hopf transition occurring at $\mathrm{Ra}=3.19 \times 10^{7}$ or a subcritical hysteretic transition between 3.3 and $3.5 \times 10^{7}$. The physical origin of the instability was not discussed in this study.

The mechanism of the transition to unsteadiness seems to be quite different for perfectly conducting horizontal walls. It was suggested in several studies, devoted to cubical cavities or to 3D perturbations in 2D base flows with a Prandtl number close to 1, that the first unsteady instability originates in a thermal instability resulting from an unstable stratification in the horizontal boundary layers. ${ }^{9,13}$ Janssen and Henkes ${ }^{13}$ have managed to recover the right magnitude of the frequency obtained from direct numerical simulations (DNS) using a simple analytical model based on the thickness of the unstable zone in the horizontal boundary layers and the resulting critical Rayleigh number. However, the 3D stability analysis of 2D base flows carried out by Xin and Le Quéré ${ }^{14}$ shows that the most unstable modes and the physical mechanism at the origin of the instability depend strongly on the fluid Prandtl number.

The effects of wall radiation on flow structure, temperature field, and heat transfer in confined natural convection problems have been recently investigated in the case of two-dimensional ${ }^{15,16}$ and three-dimensional ${ }^{17}$ geometries. When the active walls are vertical and the top and bottom walls are adiabatic, it is well established that wall radiation cools down the top horizontal wall, heats up the bottom horizontal wall, and reduces then the thermal stratification parameter in the cavity core. These effects may explain, at least partially, the discrepancies that were observed between numerical simulations ignoring radiation and experimental results. ${ }^{18,19}$ Some discrepancies remained however concerning the vertical stratification even when top and bottom wall temperature distributions are imposed in the numerical simulations equal to the measured ones. ${ }^{19}$ The full coupling between convection, conduction in the walls, and radiation exchange between the six walls of the cavity, yielded recently a good agreement between experimental measurements and numerical predictions for a Rayleigh number close to $1.5 \times 10^{9}{ }^{20}$

When the flowing medium absorbs and emits radiation, radiative transfer directly affects the local energy balance and strong effects on flow field and heat transfer may be observed as a result of changes in temperature distribution. Bdéoui and Soufiani ${ }^{21}$ and Borget et al. ${ }^{22,23}$ have studied, for instance, the effects of molecular gas radiation on the thresholds of instabilities in 2D Rayleigh-Bénard and vertical tall cavity problems. They have shown very significant delays of the instabilities due to thermal homogenization and to radiative dissipation of temperature disturbances. Several studies were recently devoted to the interaction between medium radiation and natural convection in square $2 \mathrm{D}$ cavities using either the gray gas approximation ${ }^{24,25}$ or global models for 
the infrared radiation of molecular gases. ${ }^{26-28}$ Some attempts have also been made to study 3D cubical differentially heated cavities filled with participating media but these studies were limited to the gray gas assumption. ${ }^{29-31}$ The general trends of volumetric radiation are a thickening of boundary layers and an increase of maximum velocities. However, the gray gas assumption fails generally to predict actual behavior of real gases as shown, for instance, by Refs. 22 and 26. Even qualitative trends may not be correctly captured when using this assumption. In a recent study, ${ }^{32}$ we have investigated the effects of wall and/or molecular gas radiation on steady natural convection in 3D cubical cavities filled with humid air. Steady solutions were obtained for $\mathrm{Ra}=10^{5}, 10^{6}$, and $3 \times 10^{7}$, except at this highest Rayleigh number in the case of radiating walls. Both wall and gas radiation were shown to decrease the thermal stratification in the core of the cavity and to increase the global circulation.

The aim of the present paper is to investigate the effects of wall and gas radiation on the transition to unsteady flows, and on the structure of weakly turbulent flows, beyond the transition, for differentially heated 3D cavities. As the cubical enclosure with adiabatic passive walls has been widely investigated without radiation, we adopt this configuration in the present study for comparison purposes. Although linear stability analysis with radiative transfer has been carried out for tall vertical and horizontal cavities, ${ }^{21,22}$ this approach is no longer practicable for $3 \mathrm{D}$ cavities. We adopt therefore direct numerical time integration of the Navier-Stokes equations, in the Boussinesq approximation, coupled to radiative transfer equations.

One among the difficulties encountered when molecular gas radiation is involved is the impossibility of defining a limited set of nondimensional parameters that could characterize the coupling between different phenomena. This is due to the complex structure of molecular absorption and emission spectra, and the resulting infinite number of optical thicknesses for a given length. The true dimensions of the cavity, the mean temperature level, and molar fractions of the absorbing species must be specified. We consider in this paper air with small contents of water vapor and carbon dioxide as the working fluid. This mixture is representative of natural convection in building applications. A dedicated spectral model is developed to deal with infrared $\mathrm{H}_{2} \mathrm{O}$ and $\mathrm{CO}_{2}$ radiative properties.

The remainder of the paper is organized as follows. Problem description, mathematical formulation, and numerical methods are described in Sec. II for both fluid flow and radiation modelling. The observed scenarios of transition to unsteadiness are analyzed in Sec. III for conventional pure natural convection, and for natural convection coupled to wall or molecular gas radiation. The effects of radiative transfer on flow dynamics in weakly turbulent regimes, above the transition to unsteadiness, are finally discussed in Sec. IV.

\section{PROBLEM DESCRIPTION AND MODELLING}

\section{A. Geometrical and thermal configuration}

The considered configuration, shown in Fig. 1, consists of a differentially heated cubical cavity of side $L$, filled with an air $/ \mathrm{H}_{2} \mathrm{O} / \mathrm{CO}_{2}$ gaseous mixture with molar fractions $X_{\mathrm{H}_{2} \mathrm{O}}=0.02, X_{\mathrm{CO}_{2}}=0.001$ if not otherwise specified, at atmospheric pressure, and at a mean temperature $T_{0}=300 \mathrm{~K}$. The variations of thermophysical properties due to these small contaminant molar fractions are neglected

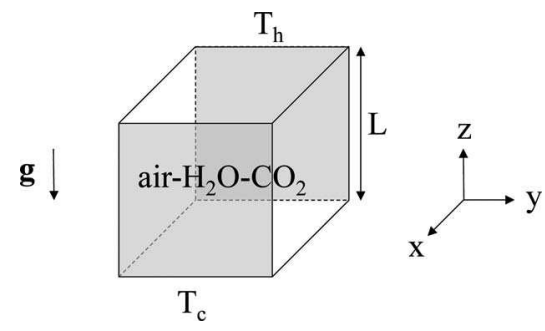

FIG. 1. Differentially heated cubical cavity. The walls $y=0, y=L, z=0$, and $z=L$ are adiabatic, while the walls $x=0$ and $x=L$ are at fixed temperatures, $T_{h}$ and $T_{c}$, respectively. 
and the Prandtl number will be taken equal to 0.71 all throughout the paper. The two opposite walls along the $x$ direction, are maintained at uniform temperatures $T_{h}=T_{0}+\Delta T / 2$ at $x=0$ and $T_{c}=T_{0}$ $-\Delta T / 2$ at $x=L$. Lateral walls, corresponding to $y=0$ and $y=L$, and also top and bottom walls are adiabatic. Concerning radiative properties, each of the six walls will be considered as perfectly diffuse, and characterized by a uniform gray emissivity $\epsilon$. Under the gravity acceleration field $\mathbf{g}=$ $-g \mathbf{e}_{z}$, the buoyancy forces induce a natural convection flow in the cavity, which may be coupled to gas radiation, due to the small amount of water vapor and carbon dioxide in the gaseous mixture, and also to wall radiation at adiabatic walls.

\section{B. Governing equations}

The dimensionless governing equations, under Boussinesq approximation are given by

$$
\begin{aligned}
\nabla \cdot \mathbf{u} & =0, \\
\frac{\partial \mathbf{u}}{\partial t}+(\mathbf{u} \cdot \nabla) \mathbf{u} & =-\nabla p+\operatorname{Pr} \theta \mathbf{e}_{z}+\frac{\operatorname{Pr}}{\sqrt{\mathrm{Ra}}} \Delta \mathbf{u}, \\
\frac{\partial \theta}{\partial t}+(\mathbf{u} \cdot \nabla) \theta & =\frac{1}{\sqrt{\mathrm{Ra}}}\left(\Delta \theta-\nabla \cdot \mathbf{q}_{R}\right),
\end{aligned}
$$

where $\mathbf{u}=(u, v, w), p$, and $\mathbf{q}_{R}$ designate the dimensionless fields of, respectively, velocity, dynamic pressure, and radiative flux, and $\theta=\left(T-T_{0}\right) /\left(T_{h}-T_{c}\right)$ is the reduced temperature. Equations (1)-(3) were made dimensionless, using the reference length $L$, the reference time $L^{2} /(a \sqrt{\mathrm{Ra}})$, and the reference flux $\lambda \Delta T / L$, where $\lambda$ and $a$ designate, respectively, the gaseous mixture thermal conductivity and diffusivity. $\mathrm{Ra}$ and $\operatorname{Pr}$ are the Rayleigh and Prandtl numbers, respectively. The associated boundary conditions are the non-slip velocity at the walls while the reduced temperature is maintained constant equal to -0.5 and 0.5 at, respectively, cold and hot walls. At any point $\mathbf{r}_{\mathrm{w}}$ of the horizontal and lateral walls, the adiabaticity condition reads

$$
\left(\mathbf{n}_{\mathrm{w}} \cdot \nabla \theta\right)\left(\mathbf{r}_{\mathrm{w}}\right)=\mathbf{n}_{\mathrm{w}} \cdot \mathbf{q}_{R}\left(\mathbf{r}_{\mathrm{w}}\right),
$$

$\mathbf{n}_{\mathrm{w}}$ being the normal to the wall at point $\mathbf{r}_{\mathrm{w}}$, directed toward the gas.

Dimensionless radiation coupling terms $\nabla \cdot \mathbf{q}_{R}$ and $\mathbf{n}_{\mathrm{w}} \cdot \mathbf{q}_{R}$ appearing in Eqs. (3) and (4) are expressed, for a medium at local thermal equilibrium, according to

$$
\begin{aligned}
\nabla \cdot \mathbf{q}_{R}(\mathbf{r}) & =\frac{L^{2}}{\lambda \Delta T}\left(4 \pi \int_{0}^{\infty} \kappa_{v} I_{b v}(T(\mathbf{r})) \mathrm{d} v-\int_{0}^{\infty} \int_{4 \pi} \kappa_{\nu} I_{\nu}(\mathbf{r}, \boldsymbol{\Omega}) \mathrm{d} \boldsymbol{\Omega} \mathrm{d} v\right) \\
\mathbf{n}_{\mathrm{w}} \cdot \mathbf{q}_{R}\left(\mathbf{r}_{\mathrm{w}}\right) & =\frac{L}{\lambda \Delta T}\left(\pi \int_{0}^{\infty} \epsilon\left(\mathbf{r}_{\mathrm{w}}\right) I_{b v}\left[T\left(\mathbf{r}_{\mathrm{w}}\right)\right] \mathrm{d} \nu-\int_{0}^{\infty} \epsilon\left(\mathbf{r}_{\mathrm{w}}\right) \int_{\boldsymbol{\Omega}^{\prime} \cdot \mathbf{n}_{\mathrm{w}}<0} I_{\nu}\left(\mathbf{r}_{\mathrm{w}}, \boldsymbol{\Omega}^{\prime}\right)\left|\boldsymbol{\Omega}^{\prime} \cdot \mathbf{n}_{\mathrm{w}}\right| \mathrm{d} \boldsymbol{\Omega}^{\prime} \mathrm{d} v\right)
\end{aligned}
$$

where $I_{\nu}(\mathbf{r}, \boldsymbol{\Omega})$ designates the spectral radiative intensity at point $\mathbf{r}$, direction $\boldsymbol{\Omega}$, and wave-number $v, I_{b v}(T)$ is the spectral equilibrium radiative intensity at temperature $T$, and $\kappa_{v}$ is the spectral gaseous absorption coefficient, which is assumed hereafter to be uniform under the Boussinesq approximation. The radiative intensity field is obtained by solving the Radiative Transfer Equation (RTE), which reads for a non-scattering medium,

$$
\boldsymbol{\Omega} \cdot \nabla I_{v}(\mathbf{r}, \boldsymbol{\Omega})=\kappa_{v}\left\{I_{b v}(T(\mathbf{r}))-I_{v}(\mathbf{r}, \boldsymbol{\Omega})\right\}
$$

associated to the boundary condition at each point $\mathbf{r}_{\mathrm{w}}$ of the six diffuse walls, for any direction $\boldsymbol{\Omega}$ satisfying $\boldsymbol{\Omega} \cdot \mathbf{n}_{\mathrm{w}}>0$

$$
I_{v}\left(\mathbf{r}_{\mathrm{w}}, \boldsymbol{\Omega}\right)=\epsilon\left(\mathbf{r}_{\mathrm{w}}\right) I_{b v}\left(T\left(\mathbf{r}_{\mathrm{w}}\right)\right)+\frac{1-\epsilon\left(\mathbf{r}_{\mathrm{w}}\right)}{\pi} \int_{\boldsymbol{\Omega}^{\prime} \cdot \mathbf{n}_{\mathrm{w}}<0} I_{\nu}\left(\mathbf{r}_{\mathrm{w}}, \boldsymbol{\Omega}^{\prime}\right)\left|\boldsymbol{\Omega}^{\prime} \cdot \mathbf{n}_{\mathrm{w}}\right| \mathrm{d} \boldsymbol{\Omega}^{\prime} .
$$

As the walls are assumed to be diffuse, the leaving intensity $I_{v}\left(\mathbf{r}_{\mathrm{w}}, \boldsymbol{\Omega}\right)$ at a given boundary point does not depend on $\boldsymbol{\Omega}$ for $\boldsymbol{\Omega} \cdot \mathbf{n}_{\mathrm{w}}>0$, and will be therefore simply denoted $I_{v}^{l}\left(\mathbf{r}_{\mathrm{w}}\right)$. Note that radiative 
intensity fields and RTE appear in dimensional form since real gas radiative properties will be considered hereafter. Only radiative source terms Eqs. (5) and (6) are made dimensionless in the flow field equations.

\section{Gas radiative property model}

In order to save computational time, a gas radiative property model based on the discretization of the absorption coefficient distribution function $(\mathrm{ADF})^{33}$ is used. This model was especially built for weak temperature gradient applications, around $T_{0}=300 \mathrm{~K}$, and for the air $/ \mathrm{H}_{2} \mathrm{O} / \mathrm{CO}_{2}$ gaseous mixture considered here. The spectral absorption coefficient of the mixture $\kappa_{\nu}$ is assumed not to vary with temperature in the small temperature range $\Delta T$ around $T_{0}$. It was calculated from a line by line approach using the EM2C database. ${ }^{34}$ In quasi-isothermal media, radiative source terms in Eqs. (5) and (6) appear as the difference between terms of similar orders of magnitude, which are much greater than their difference, as pointed out in Ref. 35. To avoid numerical inaccuracies in the computation of the net radiative source terms, an equilibrium intensity at a shift temperature $T_{s}$, slightly lower than the minimum temperature of the system, was systematically subtracted from the radiative intensities and equilibrium intensities appearing in Eqs. (7) and (8). Expressions (5) and (6) are preserved when replacing actual radiative intensities by the shifted ones. ${ }^{35}$

In the particularly simple case of a spatially uniform absorption spectrum, the ADF model just consists in substituting the wavenumber integration of radiative source terms with an integration on the value $k$ of the absorption coefficient. Note that such a global model may only be used with gray boundary properties. In practice, the $k$ range, between $k_{\min }=6.3 \times 10^{-7} \mathrm{~m}^{-1}$ and $k_{\max }=570 \mathrm{~m}^{-1}$ was logarithmically discretized in $N_{k}=16$ consecutive ranges $\left[k_{i}^{-}-k_{i}^{+}\right], i=1, \ldots, N_{k}$, each one characterized by a unique $k_{i}$ value

$$
k_{i}=\left(k_{i}^{-}\right)^{1-\alpha}\left(k_{i}^{+}\right)^{\alpha},
$$

the parameter $\alpha$ being optimized. To each scale $i$ is associated a partial shifted radiative intensity $I_{i}(\mathbf{r}, \boldsymbol{\Omega})$, which is obtained by solving the partial RTE

$$
\boldsymbol{\Omega} \cdot \nabla I_{i}(\mathbf{r}, \boldsymbol{\Omega})=k_{i}\left(\sigma \frac{T^{4}(\mathbf{r})-T_{s}^{4}}{\pi} a_{i}-I_{i}(\mathbf{r}, \boldsymbol{\Omega})\right),
$$

associated to the boundary condition for $\boldsymbol{\Omega} \cdot \mathbf{n}_{\mathrm{w}}>0$

$$
I_{i}^{l}\left(\mathbf{r}_{\mathrm{w}}\right) \equiv I_{i}\left(\mathbf{r}_{\mathrm{w}}, \boldsymbol{\Omega}\right)=\epsilon\left(\mathbf{r}_{\mathrm{w}}\right) \frac{a_{i} \sigma}{\pi}\left[T^{4}\left(\mathbf{r}_{\mathrm{w}}\right)-T_{s}^{4}\right]+\frac{1-\epsilon\left(\mathbf{r}_{\mathrm{w}}\right)}{\pi} \int_{\boldsymbol{\Omega}^{\prime} \cdot \mathbf{n}_{\mathrm{w}}<0} I_{i}\left(\mathbf{r}_{\mathrm{w}}, \boldsymbol{\Omega}^{\prime}\right)\left|\boldsymbol{\Omega}^{\prime} \cdot \mathbf{n}_{\mathrm{w}}\right| \mathrm{d} \boldsymbol{\Omega}^{\prime} .
$$

In the above equations, $\sigma$ designates the Stefan-Boltzmann constant, while the weight $a_{i}$, associated to the scale $i$, was calculated from the absorption coefficient distribution function

$$
F(k, T)=\frac{\pi}{\sigma\left(T^{4}-T_{s}^{4}\right)} \int_{v / \kappa_{v} \leq k}\left[I_{b v}(T)-I_{b v}\left(T_{s}\right)\right] \mathrm{d} v,
$$

according to $a_{i}=F\left(k_{i}^{+}, T_{0}\right)-F\left(k_{i}^{-}, T_{0}\right)$, neglecting its dependence versus temperature.

In the framework of this ADF model, the radiative source terms given in Eqs. (5) and (6) are calculated as the sum of the contributions of the different scales $i=1, \ldots, N_{k}$, according to

$$
\begin{gathered}
\nabla \cdot \mathbf{q}_{R}(\mathbf{r})=\frac{L^{2}}{\lambda \Delta T} \sum_{i=1}^{N_{k}} k_{i}\left\{4 \sigma a_{i}\left[T^{4}(\mathbf{r})-T_{s}^{4}\right]-\int_{4 \pi} I_{i}(\mathbf{r}, \boldsymbol{\Omega}) \mathrm{d} \boldsymbol{\Omega}\right\}, \\
\mathbf{n}_{\mathrm{w}} \cdot \mathbf{q}_{R}\left(\mathbf{r}_{\mathrm{w}}\right)=\frac{L \epsilon\left(\mathbf{r}_{\mathrm{w}}\right)}{\lambda \Delta T} \sum_{i=1}^{N_{k}}\left\{\sigma a_{i}\left[T^{4}\left(\mathbf{r}_{\mathrm{w}}\right)-T_{s}^{4}\right]-\int_{\boldsymbol{\Omega}^{\prime} \cdot \mathbf{n}_{\mathrm{w}}<0} I_{i}\left(\mathbf{r}_{\mathrm{w}}, \boldsymbol{\Omega}^{\prime}\right)\left|\boldsymbol{\Omega}^{\prime} \cdot \mathbf{n}_{\mathrm{w}}\right| \mathrm{d} \boldsymbol{\Omega}^{\prime}\right\} .
\end{gathered}
$$

More details on the development and accuracy of this ADF model may be found in Ref. 32. 


\section{Numerical methods}

\section{Radiative transfer}

A ray-tracing algorithm has been implemented to calculate the contribution of each ADF scale to the radiative source terms. This approach consists in generating $N_{\Omega}$ (respectively, $N_{\Omega}^{\mathrm{w}}$ ) rays from each center point $\mathbf{r}$ of volume (resp. surface) cells at which the source term $\nabla \cdot \mathbf{q}_{R}\left(\operatorname{resp} . \mathbf{n}_{\mathrm{w}} \cdot \mathbf{q}_{R}\right.$ ) has to be calculated. The direction $\boldsymbol{\Omega}$ of each ray issued from a volume cell center is defined by its zenithal $\vartheta$ and azimuthal $\varphi$ angles around the $z$ axis according to $\boldsymbol{\Omega}=\mathbf{e}_{z} \cos \vartheta+\left(\mathbf{e}_{x} \cos \varphi+\mathbf{e}_{y} \sin \varphi\right) \sin \vartheta$. Azimuthal angles $\varphi_{\ell}$ are discretized into $N_{\varphi}$ regularly spaced values between 0 and $2 \pi$, while zenithal angles are discretized according to $N_{\vartheta}$ regularly spaced $\cos \vartheta_{j}$ values between -1 and 1 , enabling one to achieve the solid angle integration in Eq. (13), using $N_{\Omega}=N_{\vartheta} \times N_{\varphi}$ directions, according to

$$
\int_{4 \pi} I_{i}(\mathbf{r}, \boldsymbol{\Omega}) \mathrm{d} \boldsymbol{\Omega}=\frac{4 \pi}{N_{\Omega}} \sum_{j=1}^{N_{\vartheta}} \sum_{\ell=1}^{N_{\varphi}} I_{i}\left(\mathbf{r}, \boldsymbol{\Omega}_{\ell j}\right) .
$$

The directions $\boldsymbol{\Omega}^{\prime}$ of rays issued from wall boundary points are similarly characterized by their zenithal $\vartheta$ and azimuthal $\varphi$ angles, but now defined around the local normal to the wall $\mathbf{n}_{\mathrm{w}} \cdot N_{\varphi}^{\mathrm{w}}$ regularly spaced $\varphi_{\ell}$ values between 0 and $2 \pi$, and $N_{\vartheta}^{\mathrm{w}}$ regularly spaced $\cos ^{2} \vartheta_{j}$ values between 0 and 1, have been used, enabling one to achieve solid angle integration in Eq. (14) following:

$$
\int_{\boldsymbol{\Omega}^{\prime} \cdot \mathbf{n}_{\mathrm{w}}<0} I_{i}\left(\mathbf{r}_{\mathrm{w}}, \boldsymbol{\Omega}^{\prime}\right)\left|\boldsymbol{\Omega}^{\prime} \cdot \mathbf{n}_{\mathrm{w}}\right| \mathrm{d} \boldsymbol{\Omega}^{\prime}=\frac{\pi}{N_{\Omega}^{\mathrm{w}}} \sum_{j=1}^{N_{\vartheta}^{\mathrm{w}}} \sum_{\ell=1}^{N_{\varphi}^{\mathrm{w}}} I_{i}\left(\mathbf{r}_{\mathrm{w}}, \boldsymbol{\Omega}_{\ell j}^{\prime}\right),
$$

with $N_{\Omega}^{\mathrm{w}}=N_{\vartheta}^{\mathrm{w}} \times N_{\varphi}^{\mathrm{w}}$.

The intensity $I_{i}(\mathbf{r}, \boldsymbol{\Omega})$ incoming at each point $\mathbf{r}$ in the direction $\boldsymbol{\Omega}$ is obtained by solving Eq. (10) along the ray ranging from the current point $\mathbf{r}$ of abscissa $s=0$ to the wall at point $\mathbf{r}_{\mathrm{w}}=\mathbf{r}-l \boldsymbol{\Omega}$ of abscissa $s=l$, according to

$$
I_{i}(\mathbf{r}, \boldsymbol{\Omega})=\int_{0}^{l} k_{i} \frac{a_{i} \sigma}{\pi}\left[T^{4}(s)-T_{s}^{4}\right] e^{-k_{i} s} \mathrm{~d} s+I_{i}^{l}\left(\mathbf{r}_{\mathrm{w}}\right) e^{-k_{i} l} .
$$

The discretization of the integrations along the rays in the above equation is easily achieved using exact intersection calculations between rays and the Cartesian mesh on which the temperature field is provided. The set of Eq. (17) expressed at each boundary point $\mathbf{r}_{\mathrm{w}}$ for the incoming intensities in each direction $\boldsymbol{\Omega}_{\ell j}^{\prime}$, together with Eqs. (11) and (16), enable us to calculate iteratively the leaving and incoming intensities at each boundary point. Equations (15) and (17) enable therefore to calculate the incoming intensity at each point of the medium, using the previously obtained leaving intensities at boundaries. The radiative source terms are then easily calculated from Eqs. (13) and (14). Validations of the above described ray-tracing algorithm were provided in a previous study by comparison with results obtained from a Monte Carlo approach, considering a 3D prescribed analytical temperature profile in a cubical enclosure. ${ }^{32}$

In practice, the ray tracing algorithm is implemented in a parallel way by distributing the $N_{\vartheta} N_{\varphi}$ and the $N_{\vartheta}^{w} N_{\varphi}^{w}$ rays among different processors. Numerical tests showed that the values $N_{\vartheta}^{w}=30$, $N_{\vartheta}=N_{\varphi}=N_{\varphi}^{w}=60$, used in the following, yield excellent accuracy.

\section{Flow field DNS solver}

The algorithm implemented to solve the natural convection flow equations Eqs. (1)-(3) is close to the one developed by Xin and Le Quéré ${ }^{36}$ and is based on a Chebyshev collocation method for the three dimensions of space. The pressure-flow coupling is ensured by a projection method, in two steps. First, momentum and energy equations are solved using the previous time step pressure field. Then, a pressure correction term is calculated from a Poisson equation and the predicted velocity is corrected in order to force the velocity divergence free condition. Time integration is performed through a second order temporal scheme combining a Backward Differentiation (BDF2) scheme for the linear terms with an Adams Bashforth extrapolation of convective terms. This 
TABLE I. Comparison between present average results and average results of Trias et al. ${ }^{38-40}$ for a rectangular cavity of aspect ratios $L_{z} / L_{x}=4, L_{y} / L_{x}=1$, and $\mathrm{Ra}_{L_{z}}=2 \times 10^{9}$.

\begin{tabular}{lcc}
\hline \hline & Our results & ${\text { Trias } \text { et al. }^{38-40}}$ \\
\hline$\overline{\mathrm{Nu}}$ & 66.55 & 66.63 \\
$\sigma\left(\mathrm{Nu}_{z}\right)_{\max }$ & 11.09 & 10.92 \\
$\bar{\theta}_{\text {top }}$ & 0.389 & 0.391 \\
$\partial \bar{\theta} / \partial z(x=0.125, z=0.5)$ & 1.02 & 1.01 \\
$\bar{w}_{\max }(x, z=0.5)$ & $2.22 \times 10^{-1}$ & $2.22 \times 10^{-1}$ \\
$\bar{u}_{\max }(x=0.125, z)$ & $1.78 \times 10^{-2}$ & $1.76 \times 10^{-2}$ \\
$\bar{\varepsilon}$ & $7.96 \times 10^{-5}$ & $8.08 \times 10^{-5}$ \\
\hline \hline
\end{tabular}

algorithm is implemented for parallel computations applying domain decomposition along the $z$ vertical direction.

Previous validations of the implementation of the pseudo-spectral solver were provided in Ref. 32, where steady solutions of the natural convection problem for different Rayleigh numbers varying from $\mathrm{Ra}=10^{5}$ to $10^{7}$, without radiation (i.e., $\nabla \cdot \mathbf{q}_{R}=0$ in the medium and $\mathbf{n}_{\mathrm{w}} \cdot \mathbf{q}_{R}=0$ at the walls) were calculated and successfully compared with the 3D benchmark solutions of Ref. 37. These previous validations are completed here by considering weakly turbulent benchmark results provided by Trias et al. ${ }^{38-40}$ in the case of a rectangular differential heated cavity of aspect ratio $L_{z} / L_{x}=4, L_{y} / L_{x}=1$, filled with air $(\operatorname{Pr}=0.71)$, without radiation, at a Rayleigh number based on the height of the cavity Ra $=2 \times 10^{9}$. Boundary conditions are the same as described in Sec. II A, except for lateral boundaries $(y=0$ and $y=L)$ where periodic boundary conditions are used for both energy and momentum equations. In this case, the pseudo-spectral solver is adapted by using a Fourier collocation method in $y$ direction and a Chebyshev collocation along $x$ and $z$ directions. The domain is decomposed into 8 identical rectangles along the $z$ axis, each one being meshed by $160 \times$ $80 \times 40$ collocation points. The dimensionless time step used is $\delta t=5 \times 10^{-4}$. These discretization features are to be compared to those used in Ref. 38, where a spatial mesh of $144 \times 64 \times 318$ points (regular along the periodic direction, and based on a hyperbolic-tangent function along the other ones), and a dimensionless time step of $\delta t=1.27 \times 10^{-3}$, were used. The initial state is that of a fluid at rest and at $\theta=0$.

Table I compares some global average quantities of the flow field, as calculated in the present study, to results obtained in Refs. 38-40. As in Refs. 38-40, the mean flow quantities are averaged here over the three statistically invariant transformations in the asymptotic state, i.e., time and $y$ direction translations, and central point symmetry around the center of the cavity. The compared quantities in Table I are the overall averaged Nusselt number at active walls $(\overline{\mathrm{Nu}})$, the maximum of the standard deviation of the local (z-dependent) averaged Nusselt number $\left(\sigma\left(\mathrm{Nu}_{z}\right)_{\max }\right)$, averaged temperature on the top wall $\left(\bar{\theta}_{\text {top }}\right)$, dimensionless stratification in the core of the cavity $(\partial \bar{\theta} / \partial z(x=$ $0.125, z=0.5)$ ), maximum value of velocity components $w$ and $u$ at, respectively, the horizontal mid-height plane $(z=0.5)$ and the vertical mid-width plane $(x=0.125)$, and the overall averaged turbulent dissipation rate, made non-dimensional according to

$$
\bar{\varepsilon}=\frac{\operatorname{Pr}}{\sqrt{\operatorname{Ra}}} \overline{\frac{\partial u_{i}^{\prime}}{\partial x_{j}} \frac{\partial u_{i}^{\prime}}{\partial x_{j}}}
$$

For all these average quantities, the differences with Refs. 38-40 do not exceed 1\%. The left part of Figure 2 shows comparisons of turbulent kinetic energy $k=\frac{1}{2} \overline{u_{i}^{\prime} u_{i}^{\prime}}$ profiles and of its dissipation $\varepsilon=\overline{\left(\partial u_{i}^{\prime} / \partial x_{j}\right)\left(\partial u_{i}^{\prime} / \partial x_{j}\right)}$ at $z=0.8$ versus $x$, while the right part of the figure shows comparisons of Nusselt number profile, and of its standard deviation, along the hot wall at $x=0$. Here also, discrepancies remain very small, and may be probably attributed to the difference of time averaging ( $\Delta t=420$ in our calculations instead of 550 in Refs. 38-40) and to the different numerical methods and meshes. 

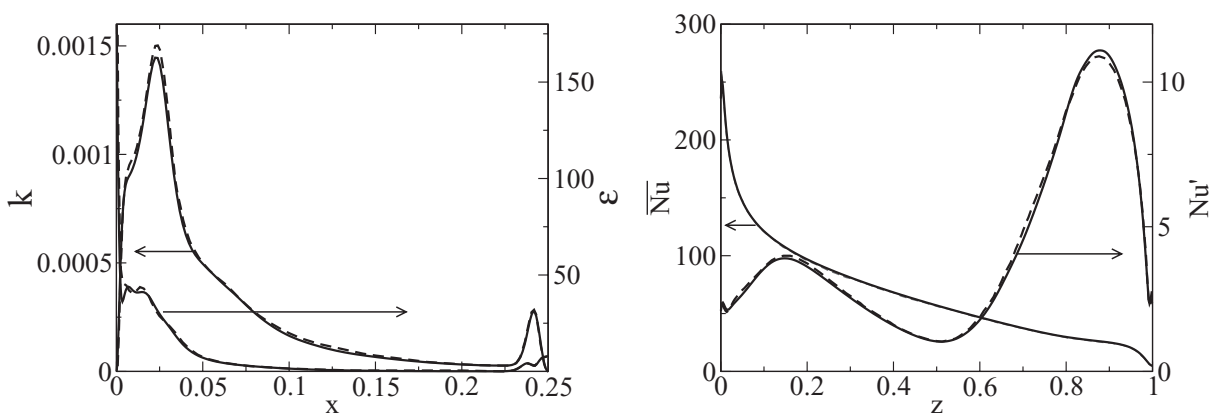

FIG. 2. Left: turbulent kinetic energy $k=0.5 \overline{u_{i}^{\prime} u_{i}^{\prime}}$ and turbulent kinetic dissipation $\varepsilon=\overline{\left(\partial u_{i}^{\prime} / \partial x_{j}\right)\left(\partial u_{i}^{\prime} / \partial x_{j}\right)}$ at $z=0.8$. Right: mean Nusselt number and its standard deviation $\mathrm{Nu}^{\prime}$ on the hot wall $x=0$. Our results in solid lines are compared to those of Trias et al. ${ }^{38,40}$ in dashed lines.

\section{Coupling with radiation}

An explicit coupling is carried out between the pseudo-spectral flow calculations described above and ray tracing radiation calculations. In practice, it is not necessary to compute radiation at each convection time step, which is mainly imposed by numerical stability constraints. The radiative source terms are updated typically every 10 convection time steps. During this period, integration of Navier-Stokes equations and radiation equations are carried out simultaneously in an asynchronous way. Radiative calculations are carried out on a coarser mesh than the convective one. The convective cells have been grouped by two in each direction for the calculations presented below to save computational time. Radiative source terms are calculated at cell centers of the coarse mesh, and are just interpolated (using Lagrange interpolation) at collocation points used to solve the energy balance equation. We have checked that the use of a 2 times coarser grid for radiation calculations enables us to capture all spatial structures of the radiative field without loss of accuracy when compared to radiation calculations carried out on the finest mesh.

\section{ONSET OF UNSTEADINESS}

The effects of wall and real gas radiation on the transition to unsteadiness are investigated considering three configurations listed in Table II. Wall and gas radiations may occur simultaneously in practical applications but it seems necessary to investigate their separate effects in order to analyze the underlying physical mechanisms. The "No-radiation" case corresponds to pure natural convection, considering a transparent medium and perfectly reflecting adiabatic walls. The isothermal walls are black but the resulting radiative flux does not affect the local energy balance or the thermal boundary conditions. In this configuration, the transition to unsteadiness, for the given Prandtl number $\operatorname{Pr}=0.71$, only depends on the Rayleigh number. In the "Wall radiation" case, the medium is assumed transparent and the six cavity walls are assumed black. Surface-surface radiative transfer modifies the thermal boundary condition on the adiabatic walls and the transition depends in this case on the Rayleigh number and on the size of the cavity $L$. In the "Gas radiation" case, the concentration of water vapor and carbon dioxide in air are accounted for and thus the medium emits and absorbs radiation. The adiabatic walls are assumed perfectly reflecting. In this last case, the transition to unsteadiness depends on the Rayleigh number, on the size of the cavity $L$ and on the

TABLE II. Studied radiation configurations.

\begin{tabular}{lccc}
\hline \hline & No-radiation & Wall radiation & Gas radiation \\
\hline Isothermal walls & $\epsilon=1$ & $\epsilon=1$ & $\epsilon=1$ \\
Adiabatic walls & $\epsilon=0$ & $\epsilon=1$ & $\epsilon=0$ \\
Medium & Transparent & Transparent & Participating \\
\hline \hline
\end{tabular}



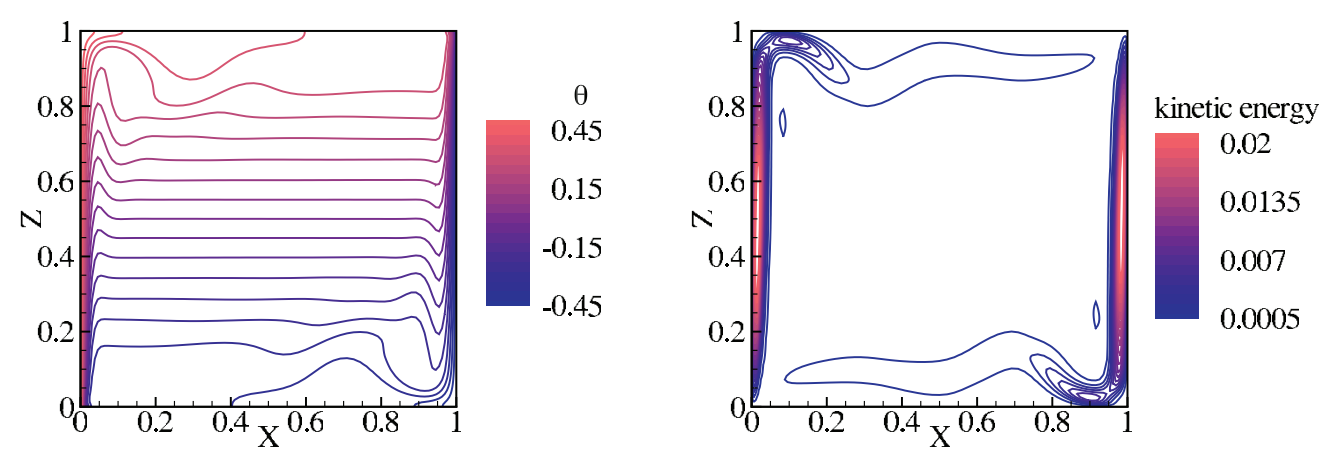

FIG. 3. No-radiation case $-\mathrm{Ra}=3.5 \times 10^{7}$. Mean temperature $\langle\theta\rangle$ and mean kinetic energy $\left\langle u_{i} u_{i} / 2\right\rangle$ in the plane $y=0.5$. The gap between two isotherms is 0.05 and the gap between two kinetic energy isovalues is $1.08 \times 10^{-3}$.

molar fractions $X_{\mathrm{H}_{2} \mathrm{O}}$ and $X_{\mathrm{CO}_{2}}$. In practice, the ratio $X_{\mathrm{H}_{2} \mathrm{O}} / X_{\mathrm{CO}_{2}}$ is kept constant equal to 20 , and the only supplementary considered parameter is $X_{\mathrm{H}_{2} \mathrm{O}}$. For the No-radiation case, we perform unsteady calculations just above the critical Rayleigh number in order to retrieve literature results. For the wall and gas radiation cases, we start from steady solutions obtained in a previous study ${ }^{32}$ and vary the transition parameters listed above until an unsteady state is reached.

\section{A. Transparent gas, perfectly reflecting adiabatic walls}

Unsteady simulation of pure natural convection is carried out at $\mathrm{Ra}=3.5 \times 10^{7}$, which corresponds to the end of the transition range $\mathrm{Ra}_{c} \in\left[3.1-3.5 \times 10^{7}\right]$ given by Labrosse et al. ${ }^{41}$ Starting from the initial state $\mathbf{u}=\mathbf{0}, p=0$ and $\theta=0$, Navier-Stokes equations are integrated using a spatial mesh made of $80 \times 80 \times(4 \times 20)$ points $(80$ Chebyshev-Gauss-Lobatto points in $x$ and $y$ directions, 20 Chebyshev-Gauss-Lobatto points in each of 4 subdomains in $z$ direction) and a time step equal to $\delta t=10^{-3}$. A total integration time equal to 2700 was required to capture several periods of the low frequency regime obtained in the asymptotic state. The final asymptotic solution is obtained from $t=1800$ with a frequency (made dimensionless by $a \sqrt{\mathrm{Ra}} / L^{2}$ ) $f=6.7 \times 10^{-3}$ close to the frequency $f=7.6 \times 10^{-3}$ found in Ref. 12 .

The mean temperature field and the mean kinetic energy field, averaged between $t=2100$ and $t=$ 2700 , are shown in Fig. 3 in the cavity mid-plane $y=0.5$. The isotherms and the flow are characterized by a sudden expansion at the exit of the vertical boundary layers. This feature has been interpreted by Ravi et al. ${ }^{41}$ as a thermal effect: the fluid oscillates because of the temperature disequilibrium at a given height between the corner region and the core. The temperature stratification $\partial \theta / \partial z$ in the stagnant core of the cavity is close to 1 . In order to extract the unsteady flow structures, the Dynamic Mode Decomposition (DMD) (see Appendix B for details) is carried out on 120 snapshots of temperature and velocity fields sampled at $\Delta t=5$. A dominant mode of amplitude one order of magnitude higher than the other's and of frequency $f=6.7 \times 10^{-3}$ is found. The real and imaginary parts of this mode are depicted in Fig. 4. The mode temperature and velocity fields show counterrotating rolls aligned along the $x$-axis, created at the exit of the vertical boundary layer and vanishing at the cavity mid-width. They display a wave-like modulation along the $y$-direction of wavelength $\lambda \simeq 0.25$ which is strongly attenuated near the lateral walls. The DMD mode retains the 2D centrosymmetry $((u, v, w, \theta)(x, y, z)=-(u,-v, w, \theta)(1-x, y, 1-z))$, but breaks the reflection symmetry relative to the mid-plane $y=0.5((u, v, w, \theta)(x, y, z)=(u,-v, w, \theta)(x, 1-y, z))$, and the 3D centrosymmetry $((u, v, w, \theta)(x, y, z)=-(u, v, w, \theta)(1-x, 1-y, 1-z))$. These counterrotating streamwise-oriented vortices have been previously observed by Janssen and Henkes. ${ }^{11}$ They performed 3D numerical simulations at $\mathrm{Ra}=10^{8}$ where they found a steady flow because of the symmetry assumptions they made to reduce the computational domain. They suggested that these rolls are caused by a centrifugal instability (Görtler instability) because of the curvature of the flow in the corner region. Similar structures have also been extracted by Xin and Le Quéré ${ }^{10}$ from a linear stability analysis of periodic perturbations along $y$-axis. 

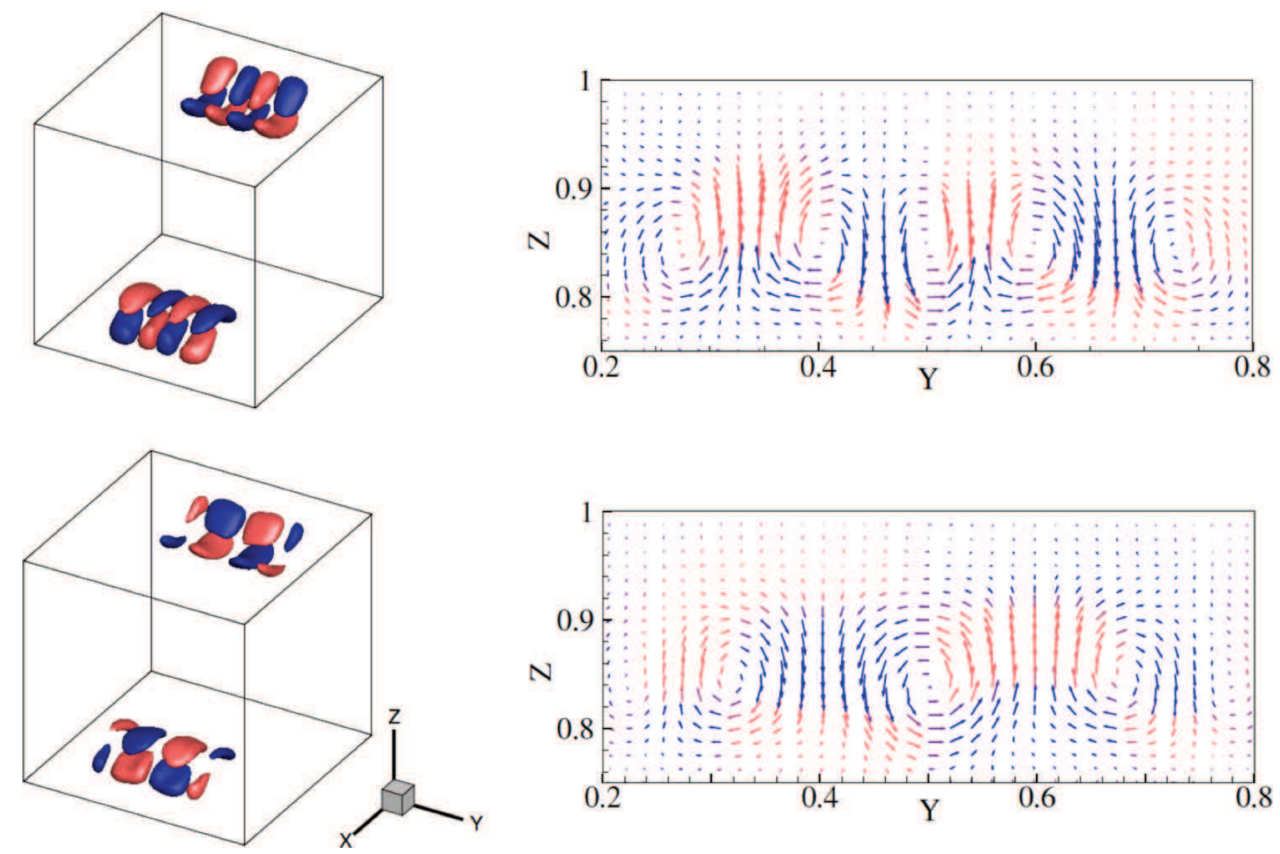

FIG. 4. No-radiation case $-\mathrm{Ra}=3.5 \times 10^{7}$. Real part (top) and imaginary part (bottom) of the dominant DMD mode of frequency $f=6.7 \times 10^{-3}$. Left: mode temperature isovalue surfaces at $\psi^{\theta}=1 \times 10^{-3}$ (lighter gray/red online) and at $\psi^{\theta}=$ $-1 \times 10^{-3}$ (darker gray/blue online); right: mode velocity vector colored by mode temperature $\psi^{\theta}$ in the plane $x=0.175$.

\section{B. Transparent gas, six black walls}

From the coupled steady solution obtained at $\mathrm{Ra}=10^{6}$ and $L=1 \mathrm{~m}$, coupled simulations of wall radiation and natural convection are made by increasing the Rayleigh number and retaining the same cavity length. At $\mathrm{Ra}=3 \times 10^{6}$, we obtain a steady solution but at $\mathrm{Ra}=5 \times 10^{6}$ we obtain an asymptotic periodic solution of frequency $f=0.22$ roughly from $t=1700$. This unsteady solution has been computed using a spatial mesh of $80 \times 80 \times(4 \times 20)$ points and a time step of $\delta t=2 \times$ $10^{-3}$.

The mean temperature field and the mean kinetic energy field, averaged between $t=1900$ and $t$ $=2300$, are shown in Fig. 5 in the cavity mid-plane $y=0.5$. As it was already noticed for the steady solution, ${ }^{32}$ the effect of wall radiation is to heat the bottom wall, to cool the top wall, and to reduce the thermal stratification at the center of the cavity. The isotherms are no more perpendicular to the adiabatic walls which leads to unstable stratification zones for $0 \leq x \leq 0.5$ near the bottom wall and for $0.5 \leq x \leq 1$ near the top wall. As the fluid is heated up (cooled down) upstream the vertical
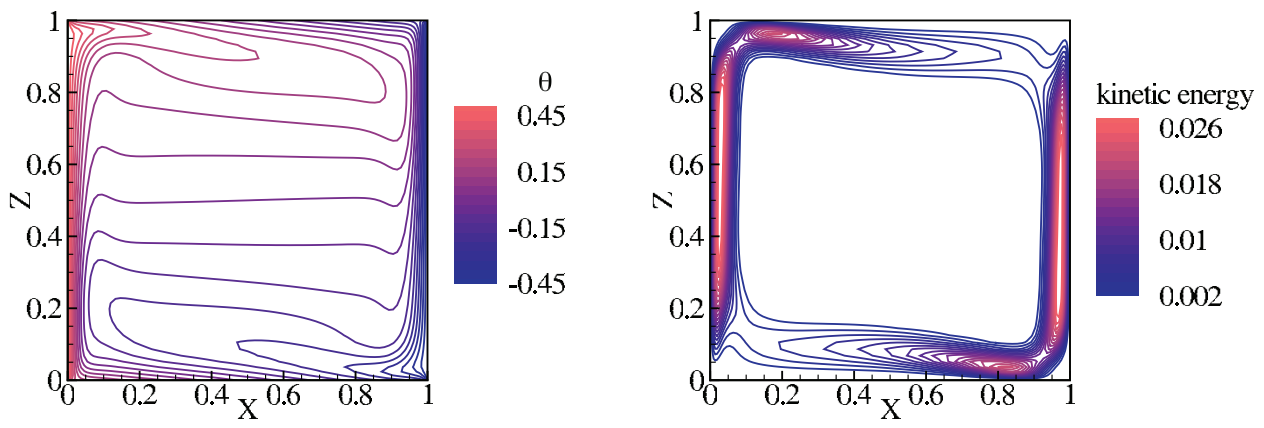

FIG. 5. Wall radiation case $-\mathrm{Ra}=5 \times 10^{6}$. Mean temperature $\langle\theta\rangle$ and mean kinetic energy $\left\langle u_{i} u_{i} / 2\right\rangle$ in the plane $y=0.5$. The gap between two isotherms is 0.05 and the gap between two kinetic energy isovalues is $1.33 \times 10^{-3}$. 

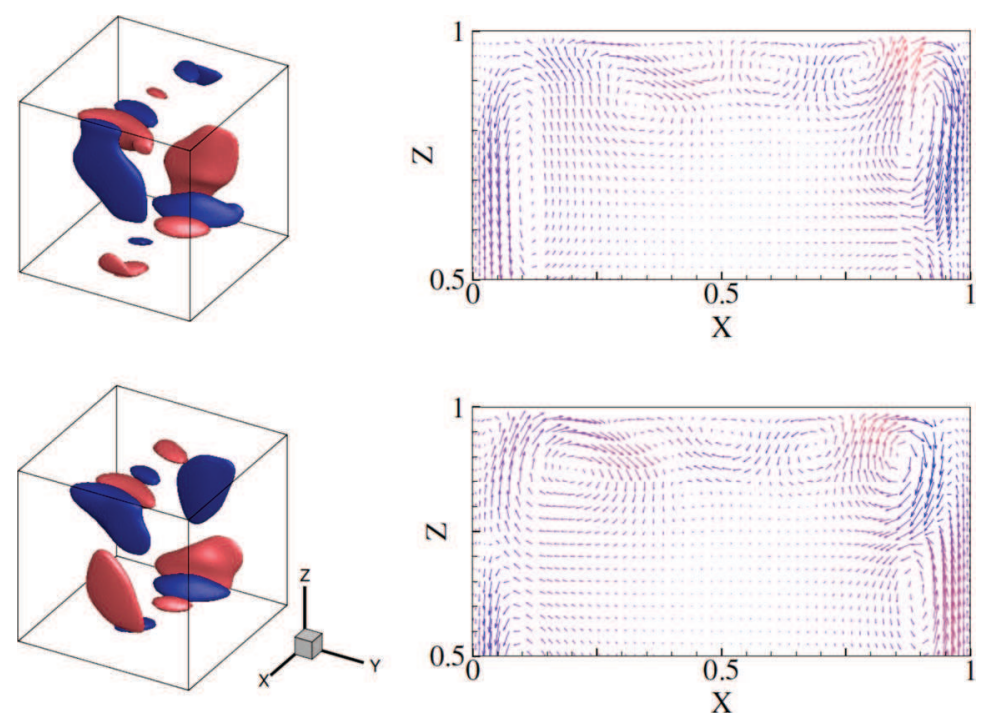

FIG. 6. Wall radiation case $-\mathrm{Ra}=5 \times 10^{6}$. Real part (top) and imaginary part (bottom) of the dominant DMD mode of frequency $f=0.22$. Left: mode temperature isovalue surfaces at $\psi^{\theta}=0.01$ (lighter gray/red online) and at $\psi^{\theta}=-0.01$ (darker gray/blue online); right: mode velocity vector colored by mode temperature $\psi^{\theta}$ in the plane $y=0.5$.

boundary layer along the hot (cold) wall, the flow driven in the cavity is increased. The typical flow expansion at the exit of the vertical boundary layers, described in the simulations without radiation, disappears because the thermal stratification is much smaller. The horizontal boundary layer is thus thinner and the velocity magnitude is higher in this region. The dynamic mode decomposition is applied on 100 snapshots of temperature and velocity fields sampled at $\Delta t=0.5$. A dominant mode of frequency $f=0.22$ is found and its real and imaginary parts are depicted in Fig. 6 . The mode temperature and velocity structures are made of rolls whose axes are perpendicular to the flow direction. These rolls grow in the unstable stratification zones before being convected in the vertical boundary layers, which suggests that they are due to a thermal instability of Rayleigh-Bénard type. The DMD mode retains the 2D centrosymmetry, but breaks the reflection symmetry relative to the mid-plane $y=0.5$ and the 3D centrosymmetry. Strictly speaking, radiative transfer should break the 2D centrosymmetry through its nonlinear dependence on the temperature field. However, the temperature differences are very small here and, as an indicator of quasi-linear radiative transfer, the mean temperature in the core of the cavity $\langle\theta(0.5,0.5,0.5)\rangle$ is about $5 \times 10^{-5}$, very close to zero. The thermal rolls grow along the top and bottom walls with roughly symmetric shapes according to the mid-plane $y=0.5$, but are stretched in the $y$-direction when they are advected in the vertical boundary layers.

In an analysis of unsteady coupled wall radiation and natural convection in 2D cavities, Wang et al. ${ }^{15}$ pointed out that radiating wall effects are similar to perfectly conducting horizontal wall effects and that the transition mechanism to unsteadiness might be similar. When the top and bottom cavity walls are perfectly conducting, their temperature profile is linear between $T_{h}$ and $T_{c}$ along $x$, which also leads to unstable stratification zones upstream the vertical boundary layers. Janssen and Henkes, ${ }^{13}$ who studied in detail the transition to unsteadiness in this configuration for $\operatorname{Pr}=0.71$, concluded that the instability mechanism is the same for $2 \mathrm{D}$ and $3 \mathrm{D}$ conducting cavities and that it is a thermal instability in the unstable stratification zones. They predicted the oscillation frequency at the onset of unsteadiness, by adapting the empirical relations of Sparrow et al. ${ }^{42}$ for "thermals" in unstable deep layers according to

$$
f=2.77^{2}\left(\frac{\Delta \theta_{\mathrm{bl}}}{\mathrm{Ra}_{\mathrm{bl}}}\right)^{2 / 3} \mathrm{Ra}^{1 / 6} .
$$

For the 2D cavity, the equivalent Rayleigh number for the horizontal boundary layers $\mathrm{Ra}_{\mathrm{bl}}$ is estimated by averaging a local equivalent Rayleigh number $\operatorname{Ra}_{b l}(x)$, based on the boundary layer 

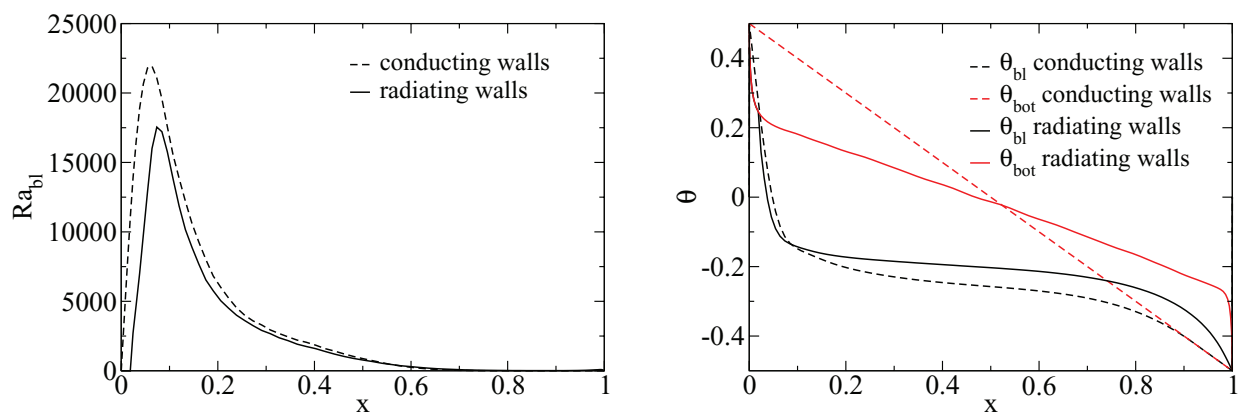

FIG. 7. Comparison between the cavity with perfectly conducting lateral and horizontal walls at $\mathrm{Ra}=3 \times 10^{6}$ and the wall radiation case at $\mathrm{Ra}=5 \times 10^{6}$. Equivalent Rayleigh number of the bottom boundary layer and profiles of the bottom wall temperature $\theta_{\mathrm{bot}}(x)$ and of the boundary layer temperature $\theta_{\mathrm{bl}}\left(x, z=\delta_{\mathrm{bl}}(x)\right)$. Results are computed from a 2D time averaged temperature field extracted at the mid-plane $y=0.5$.

thickness $\delta_{\mathrm{bl}}(x)$, defined as the temperature inversion height satisfying $\frac{\partial \theta}{\partial z}\left(x, z=\delta_{\mathrm{bl}}(x)\right)=0$, and on the temperature difference $\Delta \theta_{\mathrm{bl}}=\theta_{\mathrm{bot}}(x, z=0)-\theta_{\mathrm{bl}}\left(x, z=\delta_{\mathrm{bl}}(x)\right)$ for the bottom wall, for instance. We applied this analysis on the unsteady solution obtained at $\mathrm{Ra}=5 \times 10^{6}$ for the wall radiation case and on an unsteady solution obtained at $\mathrm{Ra}=3 \times 10^{6}$ for a cavity with perfectly conducting lateral and horizontal walls. Figure 7 compares the profiles of the equivalent Rayleigh number Rabl in the bottom boundary layer, of the bottom temperature $\theta_{\text {bot }}$ and of the boundary layer temperature $\theta_{\mathrm{bl}}$, for the two cases. The main difference relies on the temperature profile on the horizontal wall which is closer to zero in the wall radiation case. This may explain why the Rayleigh number must be higher in the wall radiation case in order to reach approximatively the same equivalent boundary layer Rayleigh number as in the case of conducting lateral walls. Equation (19) leads, with our averaged temperature field, to a nondimensional frequency $f$ equal to 0.19 , which is very close to the numerical value $f=0.22$.

The transition to unsteadiness has been studied here for a fixed cavity length. An increase of this parameter would probably decrease the critical Rayleigh number as the radiation effects would be stronger, without changing the physical transition mechanism.

\section{Radiating gas, perfectly reflecting adiabatic walls}

In a previous study, ${ }^{32}$ we obtained a steady solution for coupled gas radiation and natural convection for $\mathrm{Ra}=3 \times 10^{7}, L=1 \mathrm{~m}, X_{\mathrm{H}_{2} \mathrm{O}}=0.02$, and $X_{\mathrm{H}_{2} \mathrm{O}} / X_{\mathrm{CO}_{2}}=20$. In order to check if an unsteady regime could occur beneath the critical Rayleigh number of the No-radiation case, gas radiation effects could be made stronger by increasing either the cavity size or the participating species molar fractions. As the water vapor molar fraction should be smaller than the saturation molar fraction $X_{\mathrm{H}_{2} \mathrm{O}}^{\text {sat }}(300 \mathrm{~K})=0.0345$ at atmospheric pressure, we choose to increase the cavity size to $L=3 \mathrm{~m}$ keeping the initial concentration $X_{\mathrm{H}_{2} \mathrm{O}}=0.02$ and the Rayleigh number. Starting from the coupled steady solution obtained with $L=1 \mathrm{~m}$, an unsteady solution is found at $\mathrm{Ra}=3 \times 10^{7}$ with a strong chaotic behavior. The same computation was then carried out with the cavity size $L=$ 3 m but with lower molar fractions: an unsteady solution is found for $X_{\mathrm{H}_{2} \mathrm{O}}=0.004$ and a steady solution is found for $X_{\mathrm{H}_{2} \mathrm{O}}=0.002$. The unsteady solution obtained at $\mathrm{Ra}=3 \times 10^{7}$ for $L=3 \mathrm{~m}$ and $X_{\mathrm{H}_{2} \mathrm{O}}=0.004$ is presented here in detail as it is the closest unsteady solution to steady regimes among the configurations mentioned above. This solution has been computed using a spatial mesh of $80 \times 80 \times(4 \times 20)$ points and a time step of $\delta t=1 \times 10^{-3}$. An asymptotic unsteady regime seems to be reached around $t=200$ but without converging toward a periodic solution as it can be seen in Fig. 8 which presents the instantaneous Nusselt number at the mid-plane $x=0.5$. This Nusselt number, defined for a given plane $x$, by ${ }^{43}$

$$
\mathrm{Nu}(x, t)=\int_{y=0}^{1} \int_{z=0}^{1}\left(-\frac{\mathrm{d} \theta}{\mathrm{d} x}(x, y, z, t)+\sqrt{\mathrm{Ra}} u(x, y, z, t) \theta(x, y, z, t)\right) \mathrm{d} y \mathrm{~d} z
$$




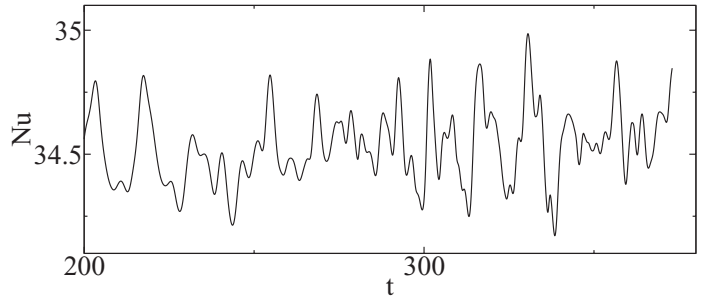

FIG. 8. Gas radiation case $-\mathrm{Ra}=3 \times 10^{7}$. Instantaneous Nusselt number $\mathrm{Nu}(x=0.5, t)$ averaged over $y$ and $z$ directions.
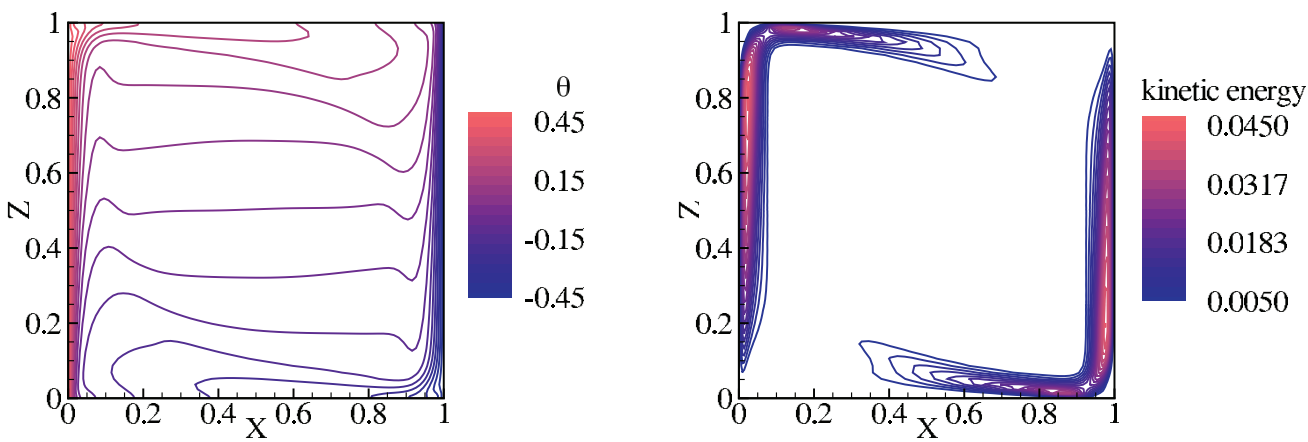

FIG. 9. Gas radiation case $-\mathrm{Ra}=3 \times 10^{7}$. Mean temperature $\langle\theta\rangle$ and mean kinetic energy $\left\langle u_{i} u_{i} / 2\right\rangle$ in the plane $y=0.5$. The gap between two isotherms is 0.05 and the gap between two kinetic energy isovalues is $2.22 \times 10^{-3}$.
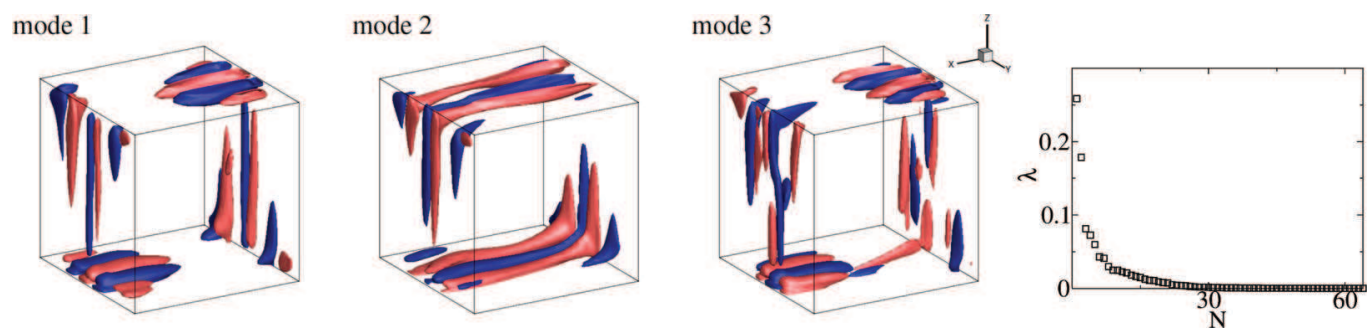

FIG. 10. Gas radiation case $-\mathrm{Ra}=3 \times 10^{7}$. Mode temperature isovalue surfaces $\psi^{\theta}= \pm 2$ of the three first POD modes and POD spectrum.

takes into account both conductive and convective fluxes, and is more sensitive to flow unsteadiness than the pure conductive Nusselt numbers at the walls. Both temperature and velocity fields display this chaotic temporal behavior.

The mean temperature field and the mean kinetic energy field, averaged between $t=200$ and $t=372$, are shown in Fig. 9 in the cavity mid-plane $y=0.5$. Gas radiation is known to decrease the thermal stratification, due to non-local gas-gas radiative exchanges, and to increase the vertical boundary layer thicknesses since the medium can be heated or cooled by radiation far from the isothermal walls. An unstable stratification near the horizontal walls does not occur here as the adiabatic walls are perfectly reflecting and thus the conductive flux on these walls is zero. It can be noticed again that the flow expansion downstream the vertical boundary layers, described for the No-radiation case, disappears due to the smaller thermal stratification. The dynamic mode decomposition does not bring here concluding results because of the temporal complexity of temperature and velocity fields. In order to capture the coherent structures of the solution, the Proper Orthogonal Decomposition (POD) (see Appendix A for details), which is based on statistical correlation between different time realizations, is preferred here. It is applied on 64 snapshots of temperature and velocity fields sampled at $\Delta t=1$. Figure 10 displays the three first POD modes and the POD spectrum. Fourteen modes are required to capture $90 \%$ of the fluctuating energy. Thermal rolls aligned along the $x$ axis can be observed on Fig. 10 for the three modes near the horizontal 


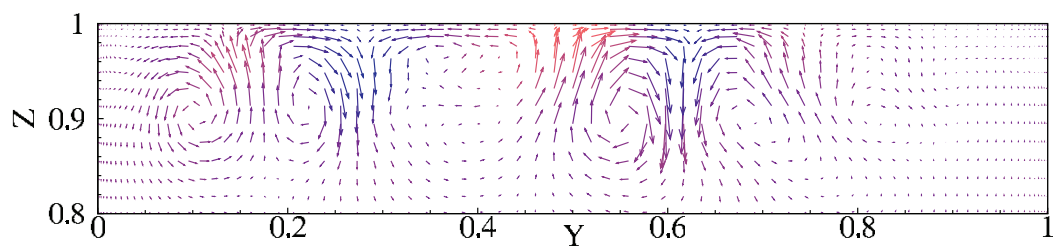

velocity fluctuations

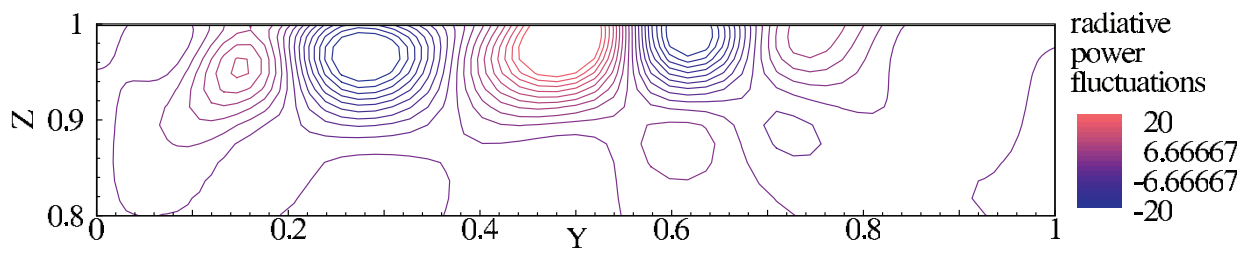

FIG. 11. Gas radiation case $-\mathrm{Ra}=3 \times 10^{7}$. Top: instantaneous velocity fluctuations colored by temperature fluctuations (lighter gray/red online for positive values and darker gray/blue online for negative values) in the plane $x=0.42$; bottom: radiative power fluctuations in the same plane.

walls but the position and the number of these rolls differ between the modes. The biggest rolls are located at the exit of the vertical boundary layer. Their size diminishes along the top and bottom walls (for modes 1 and 3 mainly) and grows again as vertical rolls along the isothermal walls. All the modes break the symmetries of the problem even if radiation can be still linearized in this case. An instantaneous velocity fluctuation field colored by the temperature fluctuation field is shown in Fig. 11 in a $x$-plane, where five counter-rotating rolls, arbitrarily located along $y$, can be seen. The corresponding radiative power $\left(\nabla \cdot \mathbf{q}_{R}\right)$ fluctuation field is also given in Fig. 11: positive radiative power fluctuations are correlated with positive temperature fluctuations while negative radiative power fluctuations are correlated with negative temperature fluctuations.

The shape of temperature POD structures and instantaneous velocity fields are closer to the structures observed in the No-radiation case than those observed in the Wall radiation case. Counterrotating rolls are clearly visible at the exit of the vertical boundary layers. Their strength decreases along the top and bottom walls but they remain active along the isothermal walls. Unlike the Noradiation case, there is no flow expansion that slows down the fluid and the fluctuations do not have time to completely disappear along the horizontal walls. These observations let us suggest that the instability mechanism is due to a hydrodynamic instability rather than a thermal instability. Like the No-radiation case, it may be caused by centrifugal forces at the exit of the vertical boundary layers. The examination of the time and volume averaged terms in the fluctuating kinetic energy balance also shows that the production term due to the mean shear $-\left\langle u_{i}^{\prime} u_{j}^{\prime}\right\rangle \frac{\partial\left\langle u_{i}\right\rangle}{\partial x_{j}}$ is about three times greater than the buoyancy production term $\operatorname{Pr}\left\langle w^{\prime} \theta^{\prime}\right\rangle$.

\section{WEAKLY TURBULENT FLOWS}

In order to study wall and gas radiation effects in a weakly turbulent regime, unsteady simulations are performed for the three configurations listed in Table II at the same Rayleigh number $\mathrm{Ra}=3 \times$ $10^{8}$, far from the onsets of unsteadiness described in Sec. III. For wall and gas radiation cases, the cavity size is fixed at $L=3 \mathrm{~m}$ and for the gas radiation case, the molar fractions of active species are fixed at $X_{\mathrm{H}_{2} \mathrm{O}}=0.02$ and $X_{\mathrm{CO}_{2}}=0.001$. The solutions have been computed using a spatial mesh of $160 \times 80 \times(8 \times 20)$ points for the No-radiation case and a spatial mesh of $160 \times 160 \times(8 \times 20)$ points for the wall and gas radiation cases. For these last two cases, the mesh was refined because stronger variations were observed in the $y$-direction. The time step is equal to $\delta t=1 \times 10^{-3}$ for the three cases.

Unsteady simulation results are analyzed here in terms of statistics on flow and thermal variables. The solution has to be integrated over a sufficiently long period in order to eliminate the transient features due to the initial condition and to obtain a large averaging time representative of the statistical average in the asymptotic regime. Starting from the initial state $\mathbf{u}=\mathbf{0}, p=0$, and 

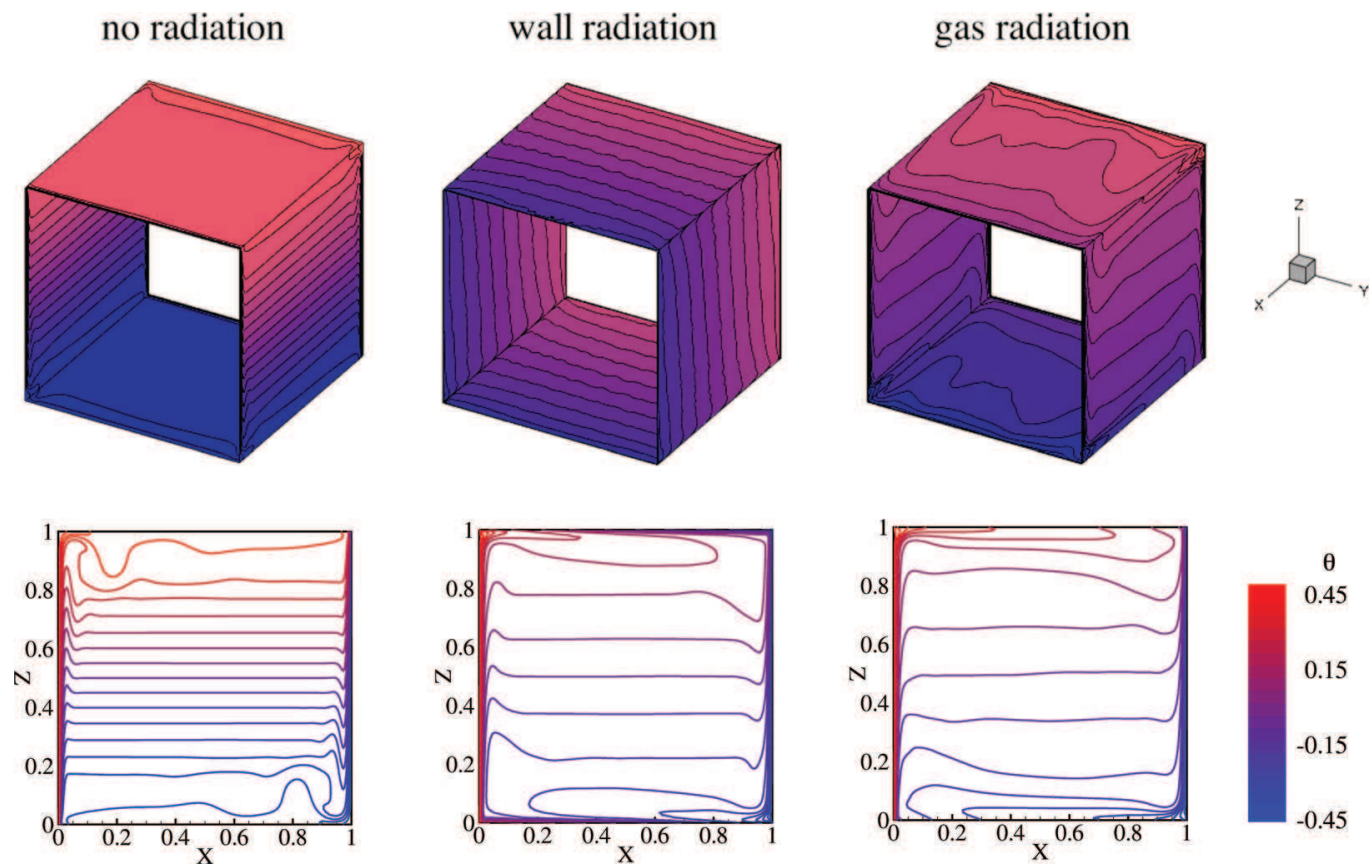

FIG. 12. Mean temperature field on the adiabatic walls (top) and in the cavity mid-plane $y=0.5$ (bottom) at $\mathrm{Ra}=3 \times 10^{8}$. The gap between two isotherms is equal to 0.05 .

$\theta=0$, the asymptotic regime for the No-radiation case is reached after $t \simeq 600$ and the results are time averaged over a period of $\Delta t=300$. Starting from the solution obtained for the No-radiation case at $t=600$, the asymptotic regime for the wall and gas radiation cases is reached after $t \simeq 300$ and the results are time averaged over a period of $\Delta t=200$. It should be pointed out that taking into account radiative transfer is very expensive in terms of computational time, compared to pure natural convection simulations. At $\mathrm{Ra}=3 \times 10^{8}$, for the gas radiation case, around $50000 \mathrm{~h} \mathrm{CPU}$ time (Intel Sandy Bridge E5-4650, $2.7 \mathrm{GHz}$ processors) are required to perform 100000 iterations $(\Delta t=100)$. The CPU time is 15 times smaller for the wall radiation case and 60 times smaller for the No-radiation case.

\section{A. Mean fields}

The mean temperature fields on the adiabatic walls and in the cavity mid-plane are depicted in Fig. 12. The temperature field features are similar to those obtained at lower Rayleigh number. When radiation is ignored, a sudden flow expansion occurs downstream the vertical boundary layers. The thermal stratification is close to 1 and the top and bottom walls are quasi-isothermal. When the six walls of the cavity are black and the gas is transparent, the temperature field on the adiabatic walls, imposed by radiation-conduction coupling, is almost linear along $x$ around the mean temperature $\theta=0$, which leads to a decrease of the stratification in the cavity core by conduction and convection transport. It can be noticed that the isotherms along the adiabatic walls are a little noisy in this case. This may be caused by the unphysical instantaneous radiation-conduction coupling on the adiabatic walls. Taking into account the wall thermal inertia, like in Ref. 20, for example, would overcome this numerical artifact. In the Gas radiation case (with black isothermal walls and perfectly reflecting adiabatic walls), the local emission and absorption of radiation homogenizes the temperature field and also strongly decreases the thermal stratification. In this configuration, the temperature distribution on the adiabatic walls follows that of the gas: the bottom (top) wall temperature is thus heated up (cooled down) compared to the No-radiation case. The temperature distribution on the vertical adiabatic walls is vertically stratified as for the No-radiation case. Fig. 12 shows that the reflection symmetry relative to the mid-plane $y=0.5$ is strongly broken on the top 


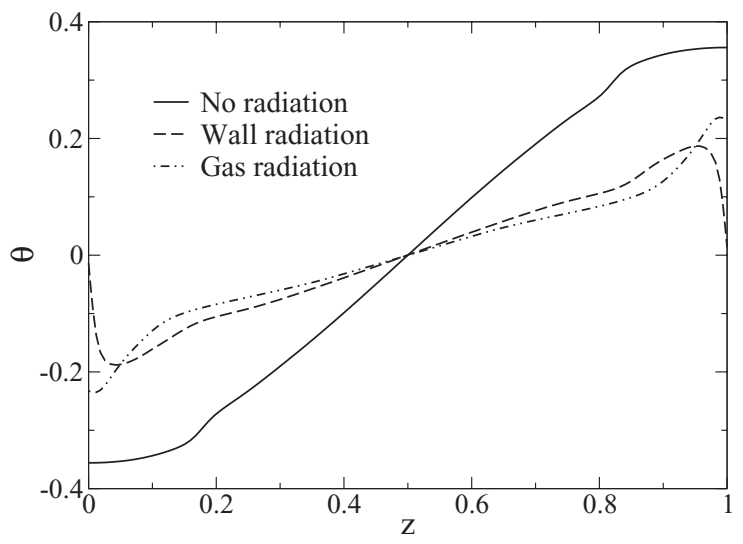

FIG. 13. Thermal stratification in the cavity core at $\mathrm{Ra}=3 \times 10^{8}$ : time averaged temperature field along the line $y=0.5, x$ $=0.5$.

and bottom walls for the Gas radiation case. This symmetry breaking is probably due to a lack of time averaging and to the low frequencies appearing in the fluctuations. The thermal stratification in the cavity core is shown more quantitatively in Fig. 13. Both wall and gas radiation strongly decrease the thermal stratification: the vertical temperature gradient at the point $(x, y, z)=(0.5,0.5,0.5)$ is equal to $1,0.4$, and 0.32 for the No-radiation, Wall radiation, and Gas radiation cases, respectively.

Figure 14 displays the time-averaged vertical component of the velocity in the vertical boundary layer near the hot wall. For the No-radiation case, the boundary layer is very thin $(\delta \simeq 0.05)$ upstream $(z=0.25)$ and exhibits a spatial oscillation at higher locations due to the flow expansion. For the wall radiation case, the boundary layer is significantly thickened upstream, because of the pre-heating of the gas along the bottom wall, and thus a larger amount of fluid is set in motion. At higher locations, the boundary layer thickness decreases and tends toward the boundary layer thickness of the Noradiation case, although the velocity profile does not oscillate because of the smaller stratification. For the gas radiation case, the velocity profile never vanishes and the cavity core is no more stagnant. The flow driven in the cavity and the $w$ maximum value are the highest in this case. More precisely, the flow driven in the cavity, calculated by averaging the vertical velocity component on a half plane at mid-height $(0 \leq x \leq 0.5,0 \leq y \leq 1, z=0.5)$, is equal to $1.11 \times 10^{-2}$ for the gas radiation case, while it is equal to $3.95 \times 10^{-3}$ and $7.11 \times 10^{-3}$ for the No-radiation and wall radiation cases, respectively. An explanation can be found regarding the radiative power profiles in Fig. 14, plotted on the same locations as the velocity profiles. The radiative power is mainly negative, except very close to the hot wall, which means that the gas is rather absorbing than emitting. It reaches a minimum value in the cavity bottom where the fluid is colder and increases at higher locations as the
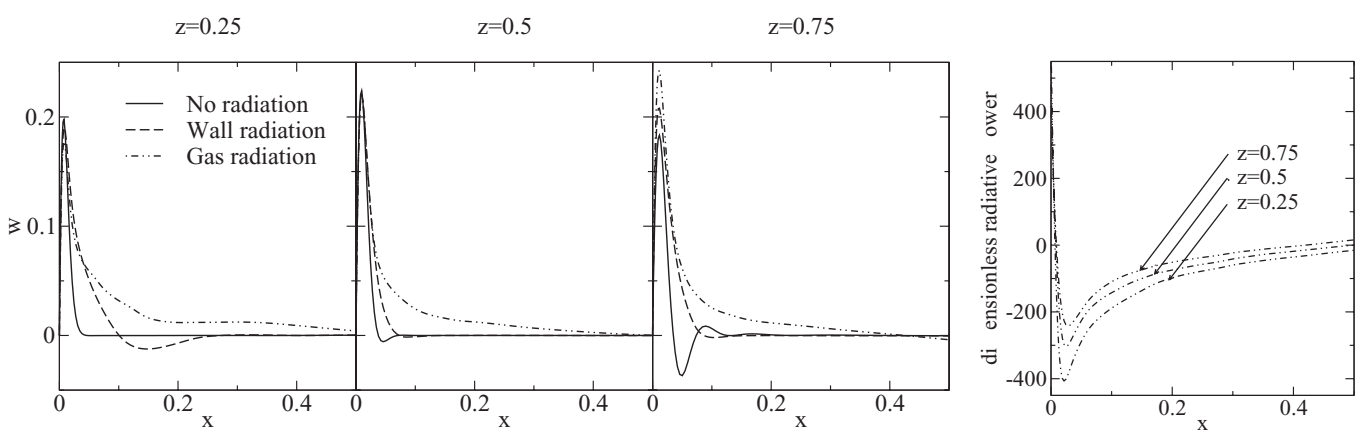

FIG. 14. Left: $w$ velocity profiles along a $x$-line at $y=0.5$ and $z=0.25, z=0.5$ and $z=0.75$ at $\mathrm{Ra}=3 \times 10^{8}$. Right: corresponding radiative power profiles for the gas radiation case. 
TABLE III. Nusselt numbers averaged on vertical planes and on time at $\mathrm{Ra}=3 \times 10^{8}$.

\begin{tabular}{lccc}
\hline \hline & No-radiation & Wall radiation & Gas radiation \\
\hline$\langle\mathrm{Nu}(x=0)\rangle$ & 39.95 & 36.52 & 35.58 \\
$\langle\mathrm{Nu}(x=0.5)\rangle$ & 39.95 & 45.07 & 73.38 \\
$\langle\mathrm{Nu}(x=1)\rangle$ & 39.95 & 36.52 & 35.58 \\
\hline \hline
\end{tabular}

wall and gas temperatures become closer. In the cavity core, wall-gas and gas-gas radiative transfers do not vanish, which activates the buoyancy forces and the fluid motion far from the active walls.

The mean Nusselt number at the isothermal walls and at the plane $x=0.5$ are also affected by wall and gas radiative transfer. They are given for the three cases in Table III. The mean Nusselt numbers at the hot and cold walls are equal in each case thanks to the mean energy conservation. As wall and gas radiation homogenize the temperature field, the averaged temperature gradients on the isothermal walls are smaller compared to the No-radiation case. For wall and gas radiation cases, the Nusselt number at $x=0.5$ is no more equal to the Nusselt number at the isothermal walls and is significantly higher than for the No-radiation case due to the increase of fluid motion.

\section{B. Second order statistics}

Table IV gives typical second order statistics averaged over the whole cavity volume: the turbulent kinetic energy $\frac{1}{2}\left\langle u_{i}^{\prime} u_{i}^{\prime}\right\rangle$, the variance of temperature fluctuations $\left\langle\theta^{\prime 2}\right\rangle$, the turbulent kinetic dissipation rate $\left\langle\left(\partial u_{i}^{\prime} / \partial x_{j}\right)\left(\partial u_{i}^{\prime} / \partial x_{j}\right)\right\rangle$, and the thermal dissipation rate $\left\langle\left(\partial \theta^{\prime} / \partial x_{i}\right)\left(\partial \theta^{\prime} / \partial x_{i}\right)\right\rangle$. All of these quantities are two orders of magnitude smaller when radiation is ignored and are the highest for the Wall radiation case even if the levels are of the same order in the Gas radiation case. Figure 15 shows the turbulent kinetic energy and the variance of temperature fluctuations in the midplane $y=0.5$ (these quantities do not strongly vary along the $y$ direction). The 2D centrosymmetry of these fields is not exactly satisfied, probably due to a lack of sufficient time averaging. For the No-radiation case, velocity and temperature fluctuations are only located on the corner at the exit of the vertical boundary layers. The shape of the isovalue surfaces looks like the structures that have been highlighted at the onset of unsteadiness. For the Wall and Gas radiation cases, velocity and temperature fluctuation maxima are one order of magnitude higher. The turbulent kinetic energy is mainly produced along the isothermal walls and is convected throughout all the cavity. Regarding the variance of temperature fluctuations, we observe that in both cases a maximum is reached at the exit of the vertical boundary layers. However, a strong temperature fluctuation production occurs on the top wall for $0.5 \leq x \leq 1$ and on the bottom walls for $0 \leq x \leq 0.5$ for the Wall radiation case, while temperature fluctuations are negligible in this region for the Gas radiation case. This region corresponds to the unstable stratification zone for the wall radiation case, which has been previously discussed.

The proper orthogonal decomposition has been carried out for each cases from 100 snapshots of velocity and temperature fields sampled at $\Delta t=1$. The POD spectrum and 3D temperature isovalue surfaces of the three first dominant modes are presented in Fig. 16. For the No-radiation

TABLE IV. Turbulent kinetic energy, variance of temperature fluctuations, turbulent kinetic dissipation, and thermal dissipation averaged over the whole volume and on time at $\mathrm{Ra}=3 \times 10^{8}$.

\begin{tabular}{lccc}
\hline \hline & No-radiation & Wall radiation & Gas radiation \\
\hline$\frac{1}{2}\left\langle u_{i}^{\prime} u_{i}^{\prime}\right\rangle$ & $4.06 \times 10^{-6}$ & $3.53 \times 10^{-4}$ & $2.54 \times 10^{-4}$ \\
$\left\langle\theta^{\prime 2}\right\rangle$ & $3.66 \times 10^{-6}$ & $4.10 \times 10^{-4}$ & $2.43 \times 10^{-4}$ \\
$\left\langle\frac{\partial u_{i}^{\prime}}{\partial x_{j}} \frac{\partial u_{i}^{\prime}}{\partial x_{j}}\right\rangle$ & $4.74 \times 10^{-2}$ & 6.52 & 3.52 \\
$\left\langle\frac{\partial \theta^{\prime}}{\partial x_{i}} \frac{\partial \theta^{\prime}}{\partial x_{i}}\right\rangle$ & $2.63 \times 10^{-2}$ & 4.86 & 1.85 \\
\hline \hline
\end{tabular}



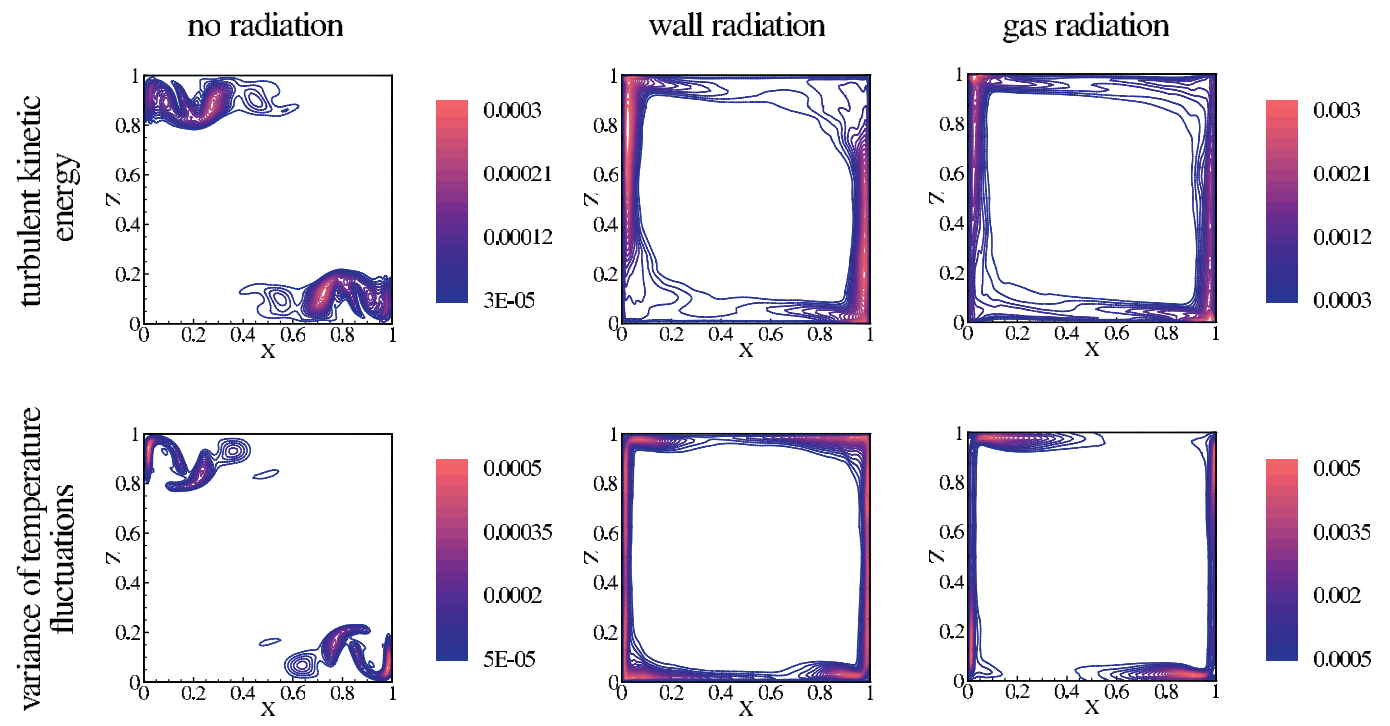

FIG. 15. Turbulent kinetic energy $\frac{1}{2}\left\langle u_{i}^{\prime} u_{i}^{\prime}\right\rangle$ (top) and variance of temperature fluctuations $\left\langle\theta^{\prime 2}\right\rangle$ (bottom) in the mid-plane $y$ $=0.5$ at $\mathrm{Ra}=3 \times 10^{8}$. For the No-radiation case, the step between two kinetic energy isovalues is $1.5 \times 10^{-5}$ and the step between two temperature variance isovalues is $2.5 \times 10^{-5}$. The same color map is used for Wall and Gas radiation cases and the corresponding scale is ten times greater than for the No-radiation case.
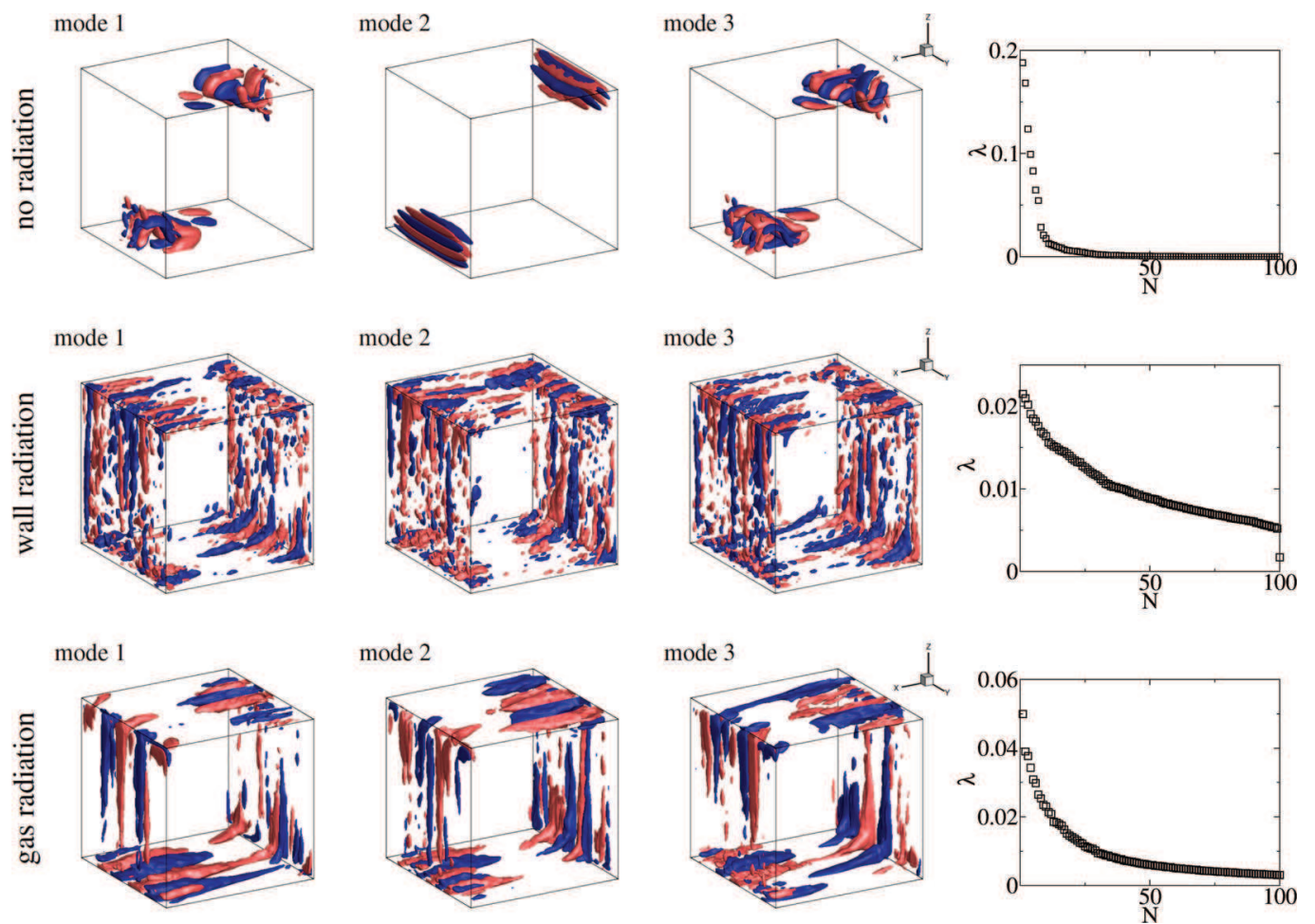

FIG. 16. Mode temperature isovalue surfaces $\psi^{\theta}= \pm 2$ of the three first POD modes and POD spectrum at $\mathrm{Ra}=3 \times 10^{8}$. 
case, the POD extracts two different types of modes: streamwise-oriented structures that oscillate along the horizontal walls (modes 1 and 3 ) and longitudinal structures along the $y$-axis typical of the boundary layer instability. These two types of modes have also been highlighted at a higher Rayleigh number by Puragliesi and Leriche, ${ }^{44}$ who performed POD in this configuration at $\mathrm{Ra}=$ $10^{9}$. The POD spectrum exhibits a fast decrease: 14 modes are sufficient here to capture $90 \%$ the energy content. On the contrary, the POD spectrum for the wall radiation case decreases slowly and the last modes still contain a significant part of the total energy. The POD modes display indeed the most chaotic behavior and are made of a large number of structures with various shapes and sizes depending on the mode. The thermal structures that were extracted at the onset of unsteadiness no longer appear probably because the intensity of the flow promotes streamwise-oriented structures. The most chaotic flow is obtained in this case because the transition to unsteadiness occurs at the lowest Rayleigh number, almost two orders of magnitude below $3 \times 10^{8}$. Finally, concerning the gas radiation case, the POD gives results close to those obtained at $\mathrm{Ra}=3 \times 10^{7}$, even if the results are more chaotic here as it can be seen from the shape of the structures and in the POD spectrum. The biggest thermal structures are still located along the horizontal walls, at the exit of the vertical boundary layers.

\section{CONCLUDING REMARKS}

Transitional regimes of natural convection in a differentially heated 3D cavity, coupled with wall or molecular gas radiation, have been investigated by means of direct numerical simulations based on accurate pseudo-spectral and ray tracing numerical methods. The aim was to study the influence of radiation on the mechanisms driving the transition to unsteadiness and to investigate radiation effects in weakly turbulent regimes. The horizontal and lateral walls were assumed to be perfectly insulated. Results with radiation have been systematically compared to pure natural convection results for which previously analyzed mechanisms have been retrieved.

In the case of radiating walls and transparent medium, surface radiation leads to an homogenization of lateral wall temperatures, especially for top and bottom walls, and the resulting transition mechanism is similar to that obtained with perfectly conducting lateral walls without radiation. The transition is due to an unstable stratification, of Rayleigh-Bénard type, upstream the vertical boundary layers and is characterized by periodically oscillating transverse rolls of axis perpendicular to the main flow. Above the transition, at $\mathrm{Ra}=3 \times 10^{8}$, wall radiation was found to significantly increase fluctuation intensities with coherent structures mainly in the form of streamwise oriented rolls.

When gas radiation, due to small contents of $\mathrm{H}_{2} \mathrm{O}$ and $\mathrm{CO}_{2}$ in air, is accounted for, the transition to unsteadiness displays complex non-periodic structures with chimneys-like rolls whose axes are also parallel to the main flow direction. The origin of this instability is probably due to centrifugal forces as was previously suggested for configurations without radiation. The flow structure with streamwise-oriented rolls was found to be persistent at higher Rayleigh numbers for which the turbulence levels were also increased in comparison with the No-radiation case.

In both Wall and Gas radiation cases, the mean thermal stratification in the cavity core is significantly reduced due to different mechanisms. For wall radiation, the top and bottom walls are, respectively, cooled and heated, which decreases the amplitude of vertical temperature variations. A direct homogenization of the temperature field in the fluid is obtained for radiating gases. The global circulation is also significantly enhanced by radiative transfer, especially for the Gas radiation case where the boundary layers are thickened due to the non-local radiative transfer.

The numerical simulations presented in this paper were carried out for specified values of the Rayleigh number, the cavity side length $L$ for wall radiation, and $L$ and molar fractions of the active species for gas radiation. While supplementary nondimensional parameters (Planck number $\mathrm{Pl}=\lambda \Delta T /\left(L \sigma T_{0}^{4}\right)$ and the ratio $\left.\Delta T / T_{0}\right)$ can be used for gray wall radiation, the treatment of gas radiation requires to take into account the so many optical thicknesses resulting from the discrete rovibrational molecular transitions. Calculations (not presented here) carried out with different molar fractions $\left(X_{\mathrm{H}_{2} \mathrm{O}}\right.$ between 0 and 0.02) and cavity side lengths (between 1 and $3 \mathrm{~m}$ ) have shown that 
the identified mechanisms of the transition to unsteadiness are not sensitive to these parameters, although the threshold Rayleigh number depends on them.

A natural continuation of this work will be the extension to higher Rayleigh numbers in order to analyze radiation effects in fully turbulent natural convection flows. This will require further models for radiation transport since direct numerical simulation of radiation, accounting for all turbulent length scales, becomes quickly prohibitive. Such models would also help to develop efficient tools for coupled radiation-large eddy simulations.

\section{ACKNOWLEDGMENTS}

Computational facilities were made available by the Institut de Developpement et des Ressources Informatiques Scientifiques (IDRIS), the CNRS Computer Center in Orsay, France. Support by the Energy Program (COCORAPHA) from CNRS is gratefully acknowledged.

\section{APPENDIX A: PROPER ORTHOGONAL DECOMPOSITION}

The POD (see Ref. 45) aims at constructing an optimal basis for a vector field $\boldsymbol{\phi}(\mathbf{r}, t)$, of zero statistical mean $\langle\boldsymbol{\phi}(\mathbf{r}, t)\rangle=0$, with regard to its energy content $\left\langle\boldsymbol{\phi}(\mathbf{r}, t)^{2}\right\rangle$. Spatial modes $\boldsymbol{\psi}_{n}(\mathbf{r})$ are sought such that they maximize the projection of $\phi(\mathbf{r}, t)$

$$
\max _{\|\boldsymbol{\psi}\|=1}\left\langle(\boldsymbol{\phi}, \boldsymbol{\psi})^{2}\right\rangle
$$

where the scalar product for two vector fields $f(\mathbf{r})$ and $g(\mathbf{r})$ and the associated norm are given by

$$
\begin{gathered}
(\boldsymbol{f}, \boldsymbol{g})=\int_{\Omega} \sum_{m=1}^{M} f^{m}(\mathbf{r}) g^{m}(\mathbf{r}) d \mathbf{r}, \\
\|\boldsymbol{f}\|=\sqrt{(\boldsymbol{f}, \boldsymbol{f})},
\end{gathered}
$$

$M$ being the dimension of the vector fields. Equation (A1) leads to the following eigenvalue problem:

$$
\int_{\Omega} \sum_{k=1}^{M}\left\langle\phi^{m}(\mathbf{r}, t) \phi^{k}\left(\mathbf{r}^{\prime}, t\right)\right\rangle \psi^{k}\left(\mathbf{r}^{\prime}\right) d \mathbf{r}^{\prime}=\lambda \psi^{m}(\mathbf{r}) .
$$

As the statistical average is estimated in practice by averaging $N$ snapshots $\phi\left(\mathbf{r}, t_{i}\right)$ extracted at discrete times $t_{i}$, Sirovich ${ }^{46}$ proposed an equivalent problem of lower dimension

$$
\begin{gathered}
\mathbf{K} \boldsymbol{\alpha}_{n}=\lambda_{n} \boldsymbol{\alpha}_{n}, \\
\mathbf{K}_{i j}=\frac{1}{N}\left(\boldsymbol{\phi}\left(\mathbf{r}, t_{i}\right), \boldsymbol{\phi}\left(\mathbf{r}, t_{j}\right)\right),
\end{gathered}
$$

where $\mathbf{K}$ is a real symmetric matrix which has real positive eigenvalues $\lambda_{n}$ and orthogonal eigenvectors $\boldsymbol{\alpha}_{n}$. The spatial modes are built by combining the eigenvectors and the snapshots according to

$$
\boldsymbol{\psi}_{n}(\mathbf{r})=\sum_{i=1}^{N} \boldsymbol{\phi}\left(\mathbf{r}, t_{i}\right) \alpha_{n}^{i} .
$$

These spatial modes form an orthonormal basis, allowing the decomposition of the signal

$$
\boldsymbol{\phi}(\mathbf{r}, t) \simeq \sum_{n=1}^{N} a_{n}(t) \boldsymbol{\psi}_{n}(\mathbf{r})
$$

Thanks to the spatial mode orthogonality, the projection coefficients $a_{n}(t)$ are statistically uncorrelated and their variance is equal to the eigenvalue $\lambda_{n}$. The signal $\phi$ can be approximated by a small number of modes with dominant eigenvalues. 
The analysis of natural convection flow involves fluctuating velocity and temperature fields which are combined according to $\boldsymbol{\phi}=\left\{\boldsymbol{u}^{\prime}, \gamma \theta^{\prime}\right\}$, where $\gamma$ is a factor (introduced, for instance, in Ref. 47) which compares the energy content of velocity and temperature fields

$$
\gamma^{2}=\left\langle\frac{\left\|\mathbf{u}^{\prime}\right\|^{2}}{\left\|\theta^{\prime}\right\|^{2}}\right\rangle
$$

Snapshots are sampled at fixed frequency over a large time interval in order to be representative of the statistical average and to capture low frequency dynamics. The number of snapshots $N$ is sufficient when almost all of the energy content of the signal is captured by a number $n$ of modes such that $n \ll N$.

\section{APPENDIX B: DYNAMIC MODE DECOMPOSITION}

The DMD (see Refs. 48 and 49) provides a decomposition of time evolving data sequence in terms of modes with temporal frequency and growth rate. The dynamic modes are the eigenvectors of a matrix A which could rely linearly two instants of a data sequence $\phi_{i} \equiv \phi\left(\mathbf{r}, t_{i}\right)$

$$
\boldsymbol{\phi}_{i+1}=\mathbf{A} \boldsymbol{\phi}_{i} .
$$

If the sequence comes from a nonlinear process, the application $\mathbf{A}$ is a linear tangent approximation of the dynamical system. Eigenvalues and eigenvectors of $\mathbf{A}$ are estimated from a sequence of $N$ snapshots sampled at a fixed period such as $t_{i}=i \Delta t$

$$
\boldsymbol{\Phi}_{2}^{N}=\left[\boldsymbol{\phi}_{2}, \ldots, \boldsymbol{\phi}_{N}\right]=\mathbf{A} \boldsymbol{\Phi}_{1}^{N-1}=\boldsymbol{\Phi}_{1}^{N-1} \mathbf{C}+\boldsymbol{r},
$$

where $\mathbf{C}$ is the companion matrix of $\mathbf{A}$ which has the same first eigenvalues, and $\boldsymbol{r}$ is non-zero residual if the snapshot $\boldsymbol{\phi}_{N}$ cannot be expressed as a linear combination of the previous ones $\boldsymbol{\phi}_{1}, \ldots, \boldsymbol{\phi}_{N-1}$. The computation of matrix $\mathbf{C}$ from Eq. (B2) leads to an overdetermined problem which is solved at the least square sense following:

$$
\mathbf{C}=\left(\left(\boldsymbol{\Phi}_{1}^{N-1}\right)^{T} \boldsymbol{\Phi}_{1}^{N-1}\right)^{-1}\left(\boldsymbol{\Phi}_{1}^{N-1}\right)^{T} \boldsymbol{\Phi}_{2}^{N} .
$$

The companion matrix is then diagonalized according to

$$
\mathbf{C}=\mathbf{T}^{-1} \Lambda \mathbf{T},
$$

where $\boldsymbol{\Lambda}_{i j}=\lambda_{i} \delta_{i j}\left(\lambda_{i} \in \mathbb{C}\right)$ is the eigenvalue matrix and $\mathbf{T}_{i j}=\lambda_{i}^{j-1}$ is the Vandermonde matrix. The dynamic modes are built by combining the eigenvectors of $\mathbf{C}$ and the snapshots

$$
\psi_{n}(\mathbf{r})=\sum_{i=1}^{N-1} \phi\left(\mathbf{r}, t_{i}\right) \mathbf{T}_{i n}^{-1}
$$

Introducing the eigenvalue decomposition (B4) in Eq. (B2) allows the approximate decomposition

$$
\boldsymbol{\phi}(\mathbf{r}, t) \simeq \sum_{n=1}^{N-1} \exp \left(\omega_{n} t\right) \boldsymbol{\psi}_{n}(\mathbf{r})
$$

with $\omega_{n}=\ln \lambda_{n} / \Delta t$. A selection of the dominant dynamic modes can be done by computing their norm $\left\|\boldsymbol{\psi}_{i}\right\|$ with Eq. (A3), for example. The temporal growth rate and temporal frequency of the modes are given, respectively, by the real and imaginary parts of $\omega_{n}$.

In this study, the data are made of spatial temperature and velocity fields $\boldsymbol{\phi}=\{\boldsymbol{u}, \theta\}$. The sampling frequency $\Delta t$ and the number of snapshots $N$ are chosen using the Nyquist criterion, according to the frequency range that should be captured.

${ }^{1}$ R. M. Goody, "The influence of radiative transfer on cellular convection," J. Fluid Mech. 1, 424-435 (1956).

${ }^{2}$ E. A. Spiegel, "The convective instability of a radiating fluid layer," Astrophys. J. 132, 716-728 (1960).

${ }^{3}$ D. W. Larson and R. Viskanta, "Transient combined laminar free convection and radiation in a rectangular enclosure," J. Fluid Mech. 78, 65-85 (1976).

${ }^{4}$ G. Lauriat, "Combined radiation-convection in gray-fluids enclosed in vertical cavities," ASME J. Heat Transfer 104, 609-615 (1982). 
${ }^{5}$ S. Paolucci and D. Chenoweth, "Transition to chaos in a differentially heated vertical cavity," J. Fluid Mech. 201, 379-410 (1989).

${ }^{6}$ S. Xin and P. Le Quéré, "Direct numerical simulations of two-dimensional chaotic natural convection in a differentially heated cavity of aspect ratio 4," J. Fluid Mech. 304, 87-118 (1995).

${ }^{7}$ P. Le Quéré and M. Behnia, "From onset of unsteadiness to chaos in a differentially heated square cavity," J. Fluid Mech. 359, 81-107 (1998).

${ }^{8}$ I. Mercader, O. Batiste, L. Ramírez-Piscina, X. Ruiz, S. Rüdiger, and J. Casademunt, "Bifurcations and chaos in single-roll natural convection with low Prandtl number," Phys. Fluids 17, 104108 (2005).

${ }^{9}$ R. A. W. M. Henkes and P. Le Quéré, "Three-dimensional transition of natural-convection flows," J. Fluid Mech. 319, 281-303 (1996).

${ }^{10} \mathrm{~S}$. Xin and P. Le Quéré, "Stability of two-dimensional (2D) natural convection flows in air-filled differentially heated cavities: 2D/3D disturbances," Fluid Dyn. Res. 44, 031419 (2012).

${ }^{11}$ R. J. A. Janssen and R. A. W. M. Henkes, "Instabilities in three-dimensional differentially-heated cavities with adiabatic horizontal walls," Phys. Fluids 8, 62-74 (1996).

${ }^{12}$ G. Labrosse, E. Tric, H. Khallouf, and M. Betrouni, "A direct (pseudo-spectral) solver of the 2D/3D Stokes problem: Transition to unsteadiness of natural-convection flow in a differentially heated cubical cavity," Numer. Heat Transfer B 31, 261-276 (1997).

${ }^{13}$ R. J. A. Janssen and R. A. W. M. Henkes, "The first instability mechanism in differentially heated cavities with conducting horizontal walls," ASME J. Heat Transfer 117, 626-633 (1995).

${ }^{14}$ S. Xin and P. Le Quéré, "Linear stability analyses of natural convection flows in a differentially heated square cavity with conducting horizontal walls," Phys. Fluids 13, 2529-2542 (2001).

${ }^{15} \mathrm{H}$. Wang, S. Xin, and P. Le Quéré, "Étude numérique du couplage de la convection naturelle avec le rayonnement de surfaces en cavité carrée remplie d'air," C. R. Méc. 334, 48-57 (2006).

${ }^{16}$ H. F. Nouanegue, A. Muftuoglu, and E. Bilgen, "Heat transfer by natural convection, conduction and radiation in an inclined square enclosure bounded with a solid wall,” Int. J. Therm. Sci. 48, 871-880 (2009).

${ }^{17}$ H. Sun, E. Chénier, and G. Lauriat, "Effect of surface radiation on the breakdown of steady natural convection flows in a square, air-filled cavity containing a centered inner body," Appl. Therm. Eng. 31, 1252-1262 (2011).

${ }^{18}$ T. Fusegi, J. M. Hyun, K. Kuwahara, and B. Farouk, "A numerical study of three-dimensional natural convection in a differentially heated cubical enclosure," Int. J. Heat Mass Transfer 34, 1543-1557 (1991).

${ }^{19}$ J. Salat, S. Xin, P. Joubert, A. Sergent, F. Penot, and P. Le Quéré, "Experimental and numerical investigation of turbulent natural convection in a large air-filled cavity," Int. J. Heat Fluid Flow 25, 824-832 (2004).

${ }^{20}$ S. Xin, J. Salat, P. Joubert, A. Sergent, F. Penot, and P. L. Quéré, "Resolving the stratification discrepancy of turbulent natural convection in differentially heated air-filled cavities. Part III: A full convection-conduction-surface radiation coupling," Int. J. Heat Fluid Flow 42, 33-48 (2013).

${ }^{21}$ F. Bdéoui and A. Soufiani, "The onset of Rayleigh-Bénard instability in molecular radiating gases," Phys. Fluids 9, 3858-3872 (1997).

${ }^{22}$ V. Borget, F. Bdéoui, A. Soufiani, and P. Le Quéré, "The transverse instability in a differentially heated vertical cavity filled with molecular radiating gases. I. Linear stability analysis," Phys. Fluids 13, 1492-1507 (2001).

${ }^{23}$ V. Borget, A. Soufiani, and P. Le Quéré, "Numerical simulation of the transverse instability in a differentially heated cavity filled with radiating gases," in Proceedings of the 13th International Heat Transfer Conference, Sydney, edited by G. de Vahl Davis, and E. Leonardi (Begell House, Redding, CT, 2006), p. 6.110.

${ }^{24}$ K. Lari, M. Baneshi, S. A. Gandjalikhan Nassab, A. Komiya, and S. Maruyama, "Combined heat transfer of radiation and natural convection in a square cavity containing participating gases," Int. J. Heat Mass Transfer 54, 5087-5099 (2011).

${ }^{25}$ F. Moufekkir, M. A. Moussaoui, A. Mezrhab, M. Bouzidi, and D. Lemonnier, "Combined double-diffusive convection and radiation in a square enclosure filled with semitransparent fluid," Comput. Fluids 69, 172-178 (2012).

${ }^{26}$ G. Colomer, R. Cònsul, and A. Oliva, "Coupled radiation and natural convection: Different approaches of the SLW model for a non-gray gas mixture,” J. Quant. Spectrosc. Radiat. Transfer 107, 30-46 (2007).

${ }^{27}$ A. Ibrahim and D. Lemonnier, "Numerical study of coupled double-diffusive natural convection and radiation in a square cavity filled with a $\mathrm{N}_{2}-\mathrm{CO}_{2}$ mixture," Int. Commun. Heat Mass Transfer 36, 197-202 (2009).

${ }^{28}$ S. Meftah, A. Ibrahim, D. Lemonnier, and A. Benbrik, "Coupled radiation and double diffusive convection in nongray air- $\mathrm{CO}_{2}$ and air- $\mathrm{H}_{2} \mathrm{O}$ mixtures in cooperating situations," Numer. Heat Transfer A 56, 1-19 (2009).

${ }^{29}$ G. Colomer, M. Costa, R. Cònsul, and A. Oliva, "Three-dimensional numerical simulation of convection and radiation in a differentially heated cavity using the discrete ordinates method," Int. J. Heat Mass Transfer 47, 257-269 (2004).

${ }^{30}$ M. N. Borjini, H. Ben Aissia, K. Halouani, and B. Zeghmati, "Effect of radiative heat transfer on the three-dimensional buoyancy flow in cubic enclosure heated from the side," Int. J. Heat Fluid Flow 29, 107-118 (2008).

${ }^{31}$ P. Kumar and V. Eswaran, "A numerical simulation of combined radiation and natural convection in a differentially heated cubic cavity," ASME J. Heat Transfer 132, 023501 (2010).

${ }^{32}$ L. Soucasse, Ph. Rivière, S. Xin, P. Le Quéré, and A. Soufiani, "Numerical study of coupled molecular gas radiation and natural convection in a differentially heated cubical cavity," Comput. Therm. Sci. 4, 335-350 (2012).

${ }^{33}$ L. Pierrot, Ph. Rivière, A. Soufiani, and J. Taine, "A fictitious-gas-based absorption distribution function global model for radiative transfer in hot gases," J. Quant. Spectrosc. Radiat. Transfer 62, 609-624 (1999).

${ }^{34}$ J. Taine and A. Soufiani, "Gas IR radiative properties: From spectroscopic data to approximate models," Adv. Heat Transfer 33, 295-414 (1999).

${ }^{35} \mathrm{~L}$. Soucasse, Ph. Rivière, and A. Soufiani, "Monte Carlo methods for radiative transfer in quasi-isothermal participating media," J. Quant. Spectrosc. Radiat. Transfer 128, 34-42 (2013).

${ }^{36}$ S. Xin and P. Le Quéré, "An extended Chebyshev pseudo-spectral benchmark for the 8:1 differentially heated cavity," Int. J. Numer. Methods Fluids 40, 981-998 (2002). 
${ }^{37}$ E. Tric, G. Labrosse, and M. Betrouni, "A first incursion into the 3D structure of natural convection of air in a differentially heated cubic cavity, from accurate numerical solutions," Int. J. Heat Mass Transfer 43, 4043-4056 (2000).

${ }^{38}$ F. X. Trias, M. Soria, A. Oliva, and C. D. Pérez-Segarra, "Direct numerical simulations of two- and three-dimensional turbulent natural convection flows in a differentially heated cavity of aspect ratio 4," J. Fluid Mech. 586, 259-293 (2007).

${ }^{39}$ F. X. Trias, A. Gorobets, M. Soria, and A. Oliva, "Direct numerical simulation of a differentially heated cavity of aspect ratio 4 with Rayleigh numbers up to $10^{11}$ - Part I: Numerical methods and time-averaged flow," Int. J. Heat Mass Transfer 53, 665-673 (2010).

${ }^{40}$ F. Trias, A. Gorobets, M. Soria, and A. Oliva, "Direct numerical simulation of a differentially heated cavity of aspect ratio 4 with Rayleigh numbers up to $10^{11}$ - Part II: Heat transfer and flow dynamics," Int. J. Heat Mass Transfer 53, 674-683 (2010).

${ }^{41}$ M. R. Ravi, R. A. W. M. Henkes, and C. J. Hoogendoorn, "On the high-Rayleigh-number structure of steady laminar natural-convection flow in a square enclosure," J. Fluid Mech. 262, 325-351 (1994).

${ }^{42}$ E. M. Sparrow, R. B. Husar, and R. J. Goldstein, "Observations and other characteristics of thermals," J. Fluid Mech. 41, 793-800 (1970).

${ }^{43}$ G. De Vahl Davis and I. P. Jones, "Natural convection in a square cavity: A comparison exercise," Int. J. Numer. Methods Fluids 3, 227-248 (1983).

${ }^{44}$ R. Puragliesi and E. Leriche, "Proper orthogonal decomposition of a fully confined cubical differentially heated cavity flow at Rayleigh number $\mathrm{Ra}=10^{9}$," Comput. Fluids 61, 14-20 (2012).

${ }^{45}$ G. Berkooz, P. Holmes, and J. L. Lumley, "The proper orthogonal decomposition in the analysis of turbulent flows," Annu. Rev. Fluid Mech. 25, 539-575 (1993).

${ }^{46}$ L. Sirovich, "Turbulence and the dynamic of coherent structures. Part I: Coherent structures," Q. Appl. Math. 45, 561-571 (1987).

${ }^{47}$ B. Podvin and P. Le Quéré, "Low-order models for the flow in a differentially heated cavity," Phys. Fluids 13, 3204-3214 (2001).

${ }^{48}$ C. W. Rowley, I. Mezić, S. Bagheri, P. Schlatter, and D. S. Henningson, "Spectral analysis of nonlinear flows," J. Fluid Mech. 641, 115-127 (2009).

${ }^{49}$ P. J. Schmid, "Dynamic mode decomposition of numerical and experimental data," J. Fluid Mech. 656, 5-28 (2010). 